COMMUNICATIONS IN

ANALYSIS AND GEOMETRY

Volume 10, Number 3, 611-646, 2002

\title{
On Complete Developable Submanifolds in Complex Euclidean Spaces ${ }^{1}$
}

\author{
HUNG-HSI WU AND FANGYANG ZHENG ${ }^{2}$
}

\section{Introduction.}

In this article, we consider the class of immersed, complete, complex submanifolds $M^{n}$ in $\mathbf{C}^{N}$ which are developable, that is, the Gauss map $\Gamma: M \rightarrow G r_{\mathbf{C}}(n, N)$ of such an $M$ into the complex Grassmannian (with $\Gamma(x)=$ the subspace parallel to the tangent space $T_{x} M$ ) is everywhere degenerate. Let $r<n$ be the dimension of the image of $\Gamma$. When $r=1$, the classical Hartman-Nirenberg cylinder theorem, and its complex analogue due to Abe ([A]), state that $M$ must be a cylinder. When $r \geq 2$, there are non-cylinder examples found by Dajczer-Gromoll [D-G], Bourgain, Wu [W], and Vitter [V]. We are interested in the case when such an $M$ is not a cylinder.

Let $\mathcal{L}$ be the Gauss foliation of such an $M$, i.e., the leaves of the holomorphic foliation $\mathcal{L}$ are the level sets of the Gauss map $\Gamma$ of $M$ which are necessarily $(n-r)$-dimensional linear subvarieties of $\mathbf{C}^{N}$ (cf. e.g., $[\mathrm{F}-\mathrm{W}]$ ). Then $M$ not being a cylinder means that these leaves are not all parallel to each other. Nevertheless, we shall show that when $r=2$ and $M^{n}$ is not a cylinder, then $M$ is the total space of a holomorphic fiber bundle over a Riemann surface, and is foliated by linear subvarieties of dimension $n-1$ each of which is the union of parallel $(n-2)$-dimensional leaves of $\mathcal{L}$ (Proposition 5 and Corollary 1 of Theorem 2 in $\S 4$ ). If $M^{n}$ is furthermore an embedded hypersurface in $\mathbf{C}^{n+1}$, then our first major result is that $M^{n}$ can be completely described in terms of two pieces of data:

a (complex) plane curve $S$ in $\mathbf{C}^{2}=\left\{\left(u_{1}, u_{2}\right)\right\}$ so that its projection $\Omega=u_{1}(S)$ into the first coordinate axis is a non-empty open subset of $\mathbf{C}$, and

$$
\text { a holomorphic map } f: \Omega \rightarrow \mathbf{C}^{n} \backslash\{0\}
$$

\footnotetext{
${ }^{1}$ Research partially supported by NSF Grants.

${ }^{2}$ The second author was also supported by an Alfred P. Sloan Fellowship.
} 
(Theorem 3 of $\S 5$ ). Note that, in [D-G], Dajczer and Gromoll gave a description of the structure of real analytic hypersurfaces with Gauss rank 2 with the exception of $M^{3} \subseteq \mathbf{R}^{4}$ having non nilpotent conullity operators. Because we deal with holomorphic objects, our Theorem 3 is much more precise, and it is worth pointing out that the proof of our theorem is not a "complexification" of theirs.

Our second major result concerns the general case when the rank restriction is removed. In that case, introduce an equivalence relation among these leaves as follows: Let $\mathcal{L}_{p}$ denote the leaf of $\mathcal{L}$ passing through $p$, then by definition, $\mathcal{L}_{p}$ is equivalent to $\mathcal{L}_{q}$ iff they are identical or parallel to each other. Denote the union of all the leaves equivalent to $\mathcal{L}_{p}$ by $\tilde{\mathcal{L}}_{p}$. The possibility arises that these $\tilde{\mathcal{L}}_{p}$ 's are submanifolds of $M$ of a fixed dimension strictly bigger than that of each $\mathcal{L}_{p}$. In that event, these $\tilde{\mathcal{L}}_{p}$ 's are cylinders which foliate $M$ and whose generators are the leaves of the original Gauss foliation $\mathcal{L}$. In the case of rank 2 , we saw above that these $\tilde{\mathcal{L}}_{p}$ 's are in fact co-dimension 1 linear subvarieties (Corollary 1 of Theorem 2 in $\S 4$ ). In an unpublished manuscript, [V], Al Vitter called such an $M$ a twisted cylin$d e r$ and raised the question of whether every complete complex developable submanifold of $\mathbf{C}^{N}$ is a twisted cylinder. In Theorem 1 of $\S 2$, we show that if the dimension $r$ of the image of the Gauss map of an n-dimensional complete developable complex submanifold of complex Euclidean space satisfies $r=n-1$ or $r \leq 4$, then $M$ is always a twisted cylinder, but that if $r=5$, then there are counterexamples (Lemma 2 in $\S 3$ ). The proofs of Theorem 1 and Lemma 2 are based on an algebraic result (Proposition 2 in §2) which is the main technical part of this article.

A key ingredient in the proofs of these results is the nilpotency of the so-called conullity operators ([A]) associated with the Gauss foliation. This nilpotency is a consequence of the completeness of the developable submanifold. We clarify this situation by proving that the nilpotency is essentially equivalent to completeness of the leaves: along each leaf of the Gauss foliation, the manifold can be extended indefinitely (Proposition 1 in $\S 1$ ).

In the last section, $\S 6$, we raise some questions related to the topological version of the cylinder theorem and the holomorphic deformability of a developable submanifold into a cylinder. We also prove that when the sectional curvature of $M^{n}$ (of the restriction metric of the usual complex Euclidean metric) is non-positive, then the completeness and the developability condition $r<n$ imply that $M$ must be a cylinder (Theorem 4).

We hope that this article will generate some interest in this special class of Euclidean submanifolds, which is rich in examples and yet very restrictive. We believe this topic deserves more attention from differential geometers as 
well as workers in topology and several complex variables.

Both Theorems 1 and 3 were inspired by the considerations in Vitter's manuscript ([V]). Leaving the precise bibliographical details to the appropriate places later in this article, we wish to thank Vitter warmly for sending us the manuscript.

\section{Preliminaries and Gauss completeness.}

Let us fix some notations. Throughout this paper, by an immersed complex submanifold $\left(M^{n}, \iota\right)$ in $\mathbf{C}^{N}$ we mean a holomorphic immersion $\iota$ from a connected complex manifold $M^{n}$ of complex dimension $n$ into $\mathbf{C}^{N} .\left(M^{n}, \iota\right)$ is said to be complete, if $\iota^{*}\left(d s^{2}\right)$ is a complete Kähler metric for the usual Euclidean metric $d s^{2}$ on $\mathrm{C}^{N}$.

Definition. An immersed complex submanifold $\left(M^{n}, \iota\right)$ in $\mathbf{C}^{N \text {. is called }}$ developable, if it admits a holomorphic foliation $\mathcal{F}$ which is developable, that is, for each leaf $F$ of $\mathcal{F}, \iota(F)$ is an open subset of a linear subvariety, and the tangent space $d \iota\left(T_{x} M\right)$ is constant for all $x \in F$. Such a foliation $\mathcal{F}$ is called a developable foliation.

Note that relative to the usual Euclidean metric $d s^{2}$ on $\mathbf{C}^{N}$, those $\iota(F)$ are totally geodesic in $\mathbf{C}^{N}$ (hence totally geodesic in $\left(M^{n}, \iota^{*}\left(d s^{2}\right)\right)$ ). However, this definition is independent of the specific choice of $d s^{2}$, it is an affine concept (in fact, a projective concept to be more precise). From now on, we will simply use the fixed Euclidean metric $d s^{2}$ on $\mathbf{C}^{N}$, and use the induced metric $\iota^{*}\left(d s^{2}\right)$ on $M$. That is, $\iota$ is considered as a holomorphic isometric immersion now.

There are two essentially equivalent ways to characterize this class of submanifolds. The first one is introduced by Chern and Kuiper ([C-K]). Let

$$
\text { II }: T M \times T M \rightarrow T M^{\perp}
$$

be the second fundamental form of $\left(M^{n}, \iota\right)$ in $\mathbf{C}^{N}$, where $T M^{\perp}$ is the normal bundle of $M$ in $\mathbf{C}^{N}$. For $x \in M$, let $\mathcal{L}_{x}=\left\{V \in T_{x} M \mid \operatorname{II}(V, \cdot) \equiv 0\right\}$, and let $\nu(x)=\operatorname{dim}\left(\mathcal{L}_{x}\right)$ and $\nu=\min \{\nu(x): x \in M\} . \nu$ is called the index of relative nullity of $M$. When $\nu>0$, they showed that in the open subset

$$
M^{\prime} \equiv\{x \in M \mid \nu(x)=\nu\}
$$

the distribution $\mathcal{L}$ is integrable and gives rise to a developable foliation, called the nullity foliation, and any developable holomorphic foliation on 
$M^{\prime}$ is a subbundle of $\mathcal{L}$. We shall adopt the usual practice of identifying an integrable distribution with the foliation it defines.

The second characterization is studied by Griffiths and Harris in [G-H] and also by Fischer and the first author [F-W] using the Gauss map

$$
\Gamma: M \rightarrow G r_{\mathbf{C}}(n, N)
$$

into the complex Grassmannian. It is defined by $\Gamma(x)=d \iota\left(T_{x} M\right)$, which is identified with the $n$-dimensional complex vector subspace of $\mathbf{C}^{N}$ by parallel translation.

For $x \in M$, let $r(x)$ be the (complex) rank of $d \Gamma_{x}$, and call $r=$ $\max \{r(x): x \in M\}$ the Gauss rank of $M$. Since the differential $d \Gamma$ can be identified with the second fundamental form II of $M, \nu(x)+r(x)=n$ for all $x \in M$ (cf. e.g., [F-W]), and the nullity foliation $\mathcal{L}$ is just the kernel foliation of $d \Gamma$. For this reason, we shall also call $\mathcal{L}$ the Gauss foliation or Gauss ruling (a foliation on $M$ is called a ruling if its leaves are mapped by $\iota$ onto open subsets of linear subvarieties). We shall call $M^{\prime}$ the Gauss domain.

In particular, from these descriptions we know that if $\left(M^{n}, \iota\right)$ in $\mathbf{C}^{N}$ is developable, then it has a degenerate Gauss map, i.e., its Gauss rank $r<n$, and conversely, if $r<n$, then at least the open dense subset $M^{\prime}$ is developable. Here $M^{\prime}=M \backslash \mathcal{S}$, where $\mathcal{S}$ is the complex analytic subvariety of $M$ where $\operatorname{rank}(d \Gamma)<r$.

Next, let us recall the notion of conullity operators introduced by Abe ([A]). It is also called splitting tensor in some literature. We refer the readers to $[D-G, \S 1]$ for an excellent account of this tensor.

For an immersed complex submanifold $\left(M^{n}, \iota\right)$ in $\mathbf{C}^{N}$ with $r<n$, with the usual Euclidean metric $d s^{2}$ on $\mathbf{C}^{N}$ and let $g=\iota^{*}\left(d s^{2}\right)$, denote by $\mathcal{L}_{x}^{\perp}$ the orthogonal complement of $\mathcal{L}_{x}$ in $T_{x} M$. Then $\mathcal{L}^{\perp}$ is a rank $r$ complex subbundle of $T M$ in $M^{\prime}$. Let $T M^{\mathbf{R}}=\mathcal{L}^{\mathbf{R}} \oplus \mathcal{L}^{\perp \mathbf{R}}$ be the corresponding real spaces, and let $Z=Z^{\mathcal{L}^{\mathrm{R}}} \oplus Z^{\mathcal{L}^{\perp \mathrm{R}}}$ be the corresponding orthogonal decomposition for any $Z \in T M^{\mathbf{R}}$.

For any $X \in \mathcal{L}_{x}^{\mathbf{R}}, x \in M^{\prime}$, define the conullity operator $C_{X}: \mathcal{L}_{x}^{\perp \mathbf{R}} \rightarrow$ $\mathcal{L}_{x}^{\perp \mathbf{R}}$ by

$$
C_{X}(Y)=-\left(\nabla_{Y} \tilde{X}\right)^{\perp}, \quad Y \in \mathcal{L}_{x}^{\perp \mathbf{R}}
$$

where $\nabla$ is the covariant differentiation in $\left(\mathbf{C}^{N}, d s^{2}\right)$, and $\tilde{X}$ is any local section of $\mathcal{L}^{\mathbf{R}}$ with $\tilde{X}_{x}=X$. It is a well defined tensor field, which in addition satisfies the equation (see $[\mathrm{A}]$ )

$$
\nabla_{X_{1}} C_{X_{2}}=C_{X_{2}} \circ C_{X_{1}} \quad \forall X_{1}, X_{2} \in \mathcal{L}_{x}^{\mathbf{R}},
$$


and is symmetric with respect to the second fundamental form of $M$ :

$$
\mathrm{II}\left(C_{X}(Y), Z\right)=\operatorname{II}\left(Y, C_{X}(Z)\right), \quad \forall X \in \mathcal{L}_{x}^{\mathbf{R}}, \forall Y, Z \in \mathcal{L}_{x}^{\perp \mathbf{R}}
$$

Furthermore, $C_{X} \circ J=J \circ C_{X}, \forall X$, where $J$ is the almost complex structure. So $C_{X}$ respects the type of a tangent vector and induces a complex linear map from $\mathcal{L}_{x}^{\perp}$ to $\mathcal{L}_{x}^{\perp}$. We still denote it by $C_{U}$, with $U \in \mathcal{L}_{x}$ now.

When $\left(M^{n}, \iota^{*}\left(d s^{2}\right)\right)$ is complete, along any geodesic line $\gamma(t)$ contained in a leaf of $\mathcal{L}$, write $T=\gamma^{\prime}(t)$. Then $C_{T}$ satisfies the matrix Riccati equation $\nabla_{T} C_{T}=\left(C_{T}\right)^{2}$. A simple deduction then shows that $C_{T}$ cannot have any non-zero real eigenvalue. The commutativity of $C_{T}$ and $J$ also implies that it can not have any non-zero complex eigenvalue either, so that $C_{T}$ (or $\left.C_{T-\sqrt{-1} J T}\right)$ is always nilpotent. This result is due to Abe ([A]), and we shall refer it as Abe's nilpotency theorem from now on. See also p.3-4 of [D-R] for a more transparent proof. We note in passing that this nilpotency assertion fails in the real case even when real analyticity is assumed; see Lemma 3 in $\S 3$.

In the following, we shall show that a partial converse of the nilpotency theorem holds. That is, when the conullity operators are all nilpotent, $M$ is Gauss complete, in the following sense.

Definition. An immersed complex submanifold $\left(M^{n}, \iota\right)$ in $\mathbf{C}^{N}$ with Gauss rank $r<n$ is called Gauss complete, if for any $x \in M^{\prime}$, there exists a neighborhood $U$ of $x$ such that $\iota(U)$ is an open subset of a holomorphic immersion $\sigma: B_{\epsilon}^{r} \times \mathbf{C}^{n-r} \rightarrow \mathbf{C}^{N}$, where $B_{\epsilon}^{r}$ denotes the ball of radius $\epsilon$ in $\mathbf{C}^{r}$, so that $\left(B_{\epsilon}^{r} \times \mathbf{C}^{n-r}, \sigma\right)$ is a developable submanifold and its Gauss foliation is defined by the leaves $\{p\} \times \mathbf{C}^{n-r}$ for each $p \in B_{\epsilon}^{r}$.

In other words, Gauss completeness means the leaves of the Gauss foliation are de facto complete because, locally at least, they can be extended linearly along the $\mathcal{L}$ direction to give a developable submanifold whose Gauss foliation consists of complete linear varieties. The following partial converse to Abe's nilpotency theorem says that, in the $\mathcal{L}$ direction, the nilpotency of the conullity operators is exactly what completeness can offer.

Proposition 1. Suppose an immersed complex submanifold $\left(M^{n}, \iota\right)$ in $\mathbf{C}^{N}$ has Gauss rank $r<n$. Then $M$ is Gauss complete if and only if the conullity operators are all nilpotent.

Proof. The "only if" part is just Abe's nilpotency theorem, so we need only to prove the "if" part. Fix a point $x \in M^{\prime}$. Choose a small neighborhood 
$U$ of $x$ in $M^{\prime}$ so that $\left.\iota\right|_{U}$ is an embedding. Suppose $V=\iota(U)$ is defined by

$$
z_{\mu}=f^{\mu}\left(z_{1}, \ldots, z_{n}\right), \quad \mu=n+1, \ldots, N
$$

where $\left(z_{1}, \ldots, z_{N}\right)$ is an unitary complex linear coordinate system of $\mathbf{C}^{N}$ with $T_{\iota(x)} V$ spanned by $e_{1}, \ldots, e_{n}$, where $e_{j}=\partial / \partial z_{j}$ for $j=1, \ldots, n$.

Without loss of generality, let us assume $\iota(x)=0$ and $d \iota\left(\mathcal{L}_{x}\right)$ is spanned by $e_{r+1}, \ldots, e_{n}$. Let $B_{\epsilon}^{r} \subseteq \mathbf{C}^{r}$ be the ball of radius $\epsilon$ centered at the origin. For $u \in B_{\epsilon}^{r}$, define

$$
\phi\left(u_{1}, \ldots u_{r}\right)=\left(u_{1}, \ldots, u_{r} ; 0, \ldots, 0 ; \ldots, f^{\mu}(u, 0), \ldots\right)
$$

Thus $\phi\left(B_{\epsilon}^{r}\right) \subset V$ for all sufficiently small $\epsilon$. Fix such an $\epsilon$, then there is a holomorphic frame of $d \iota(\mathcal{L})$ along $\phi\left(B_{\epsilon}^{r}\right)$ of the form:

$$
\xi^{i}(u)=\left(g^{i 1}(u), \ldots, g^{i r}(u) ; 0, \ldots, 1, \ldots, 0 ; \ldots, h^{i \mu}(u), \ldots\right)
$$

where $i=r+1, \ldots, n$. We may further assume that $V$ is a union of open balls each of which lies in a leaf of $\mathcal{L}$ which intersect $\phi\left(B_{\epsilon}^{r}\right)$. Since the tangent space of $V$ is constant along each $\mathcal{L}_{x}$ (now regarded as a linear subvariety of $\mathbf{C}^{N}$ ), the $\left\{\xi^{i}\right\}$ extend by (Euclidean) parallel translation along each $\mathcal{L}_{x}$ to a frame field of $\mathcal{L}$ in $V$. Similarly, if $\phi_{, \beta}$ denotes $\partial \phi / \partial u_{\beta}$ for $1 \leq \beta \leq r$, then $\left\{\phi_{, \beta}\right\}$ extend by parallel translation along each $\mathcal{L}_{x}$ to be vector fields tangent to $V$. Clearly, $\left\{\phi_{, \beta}\right\}$ and $\left\{\xi^{i}\right\}$ form a holomorphic frame field of $T V$ in $V$ for $1 \leq \beta \leq r, r+1 \leq j \leq n$. Thus for $\alpha=1, \ldots, r$, each $\xi_{, \alpha}^{i}$ is a linear combination of $\left\{\phi_{, \beta}\right\}$ and $\left\{\xi^{i}\right\}$, say

$$
\begin{aligned}
\xi_{, \alpha}^{i} & =\sum_{\beta} A^{\beta} \phi_{, \beta}+\sum_{i} B_{i} \xi^{i} \\
& =\left(A^{1}, \ldots, A^{r} ; 0, \ldots, 0 ; *, \ldots, *\right)+\left(*, \ldots, * ; B_{r+1}, \ldots, B_{n} ; *, \ldots, *\right) \\
& =\left(*, \ldots, * ; B_{r+1}, \ldots, B_{n} ; *, \ldots, *\right)
\end{aligned}
$$

However, a direct computation with $\left\{\xi^{i}\right\}$ shows that

$$
\xi_{, \alpha}^{i}=\left(g_{, \alpha}^{i 1}, \ldots, g_{, \alpha}^{i r} ; 0, \ldots, 0 ; *, \ldots, *\right)
$$

where $g_{, \alpha}^{i 1}=\partial g^{i 1} / \partial u_{\alpha}$, etc. This is possible only if $B_{r+1}=\ldots=B_{r}=0$, so that

$$
\begin{aligned}
\xi_{, \alpha}^{i} & =\sum_{\beta} A^{\beta} \phi_{, \beta} \\
& =\left(A^{1}, \ldots, A^{r} ; 0, \ldots, 0 ; *, \ldots, *\right) .
\end{aligned}
$$


Thus $A^{\beta}=g_{, \alpha}^{i \beta}$ for each $\beta=1, \ldots, r$ and so

$$
\xi_{, \alpha}^{i}=\sum_{\beta=1}^{r} g_{, \alpha}^{i \beta} \phi_{, \beta}, \quad \text { for } 1 \leq \alpha \leq r, \quad \text { and } r+1 \leq i \leq n
$$

Now consider $F(u, t)=\phi(u)+\sum_{i=r+1}^{n} t_{i} \xi^{i}(u)$, where $u \in B_{\epsilon}^{r}$ and $t \in \mathbf{C}^{n-r}$. For small $u$ and $t,\{u, t\}$ define holomorphic coordinates of $V$ near 0 , with

$$
F_{*}\left(\frac{\partial}{\partial t_{i}}\right)=\xi^{i}, \quad F_{*}\left(\frac{\partial}{\partial u_{\alpha}}\right)=\left(\delta_{\alpha \beta}+\sum t_{i} g_{, \alpha}^{i \beta}\right) \phi_{, \beta} .
$$

Let $Z_{\alpha}=\left(F_{*}\left(\partial / \partial u_{\alpha}\right)\right)^{\perp}$ be the orthogonal projection of $F_{*}\left(\partial / \partial u_{\alpha}\right)$ on $\mathcal{L}^{\perp}$. Note that if $Z^{\prime}=F_{*}\left(\partial / \partial u_{\alpha}\right)-Z_{\alpha}$, then $Z^{\prime}$ is tangent $\mathcal{L}$, so that by the constancy of $\xi^{i}$ along each leaf $\mathcal{L}_{x}, \nabla_{Z^{\prime}} \xi^{i}=0$ for each $i$.

That is to say,

$$
\nabla_{F_{*}\left(\partial / \partial u_{\alpha}\right)} \xi^{i}=\nabla_{Z_{\alpha}} \xi^{i}
$$

for each $i$. Hence

$$
C_{\xi^{i}}\left(Z_{\alpha}\right)=-\left(\nabla_{Z_{\alpha}} \xi^{i}\right)^{\perp}=-\left(\nabla_{F_{*}\left(\frac{\partial}{\partial u_{\alpha}}\right)} \xi^{i}\right)^{\perp}=-\left(\xi_{, \alpha}^{i}\right)^{\perp}=-\sum g_{, \alpha}^{i \beta} Z_{\beta},
$$

which implies

$$
-\sum_{i, \beta} t_{i} g_{, \alpha}^{i \beta} Z_{\beta}=C_{\sum_{i} t_{i} \xi^{i}}\left(Z_{\alpha}\right)
$$

Therefore

$$
\bigwedge_{\alpha} F_{*}\left(\frac{\partial}{\partial u_{\alpha}}\right)=\operatorname{det}\left(I-C_{\sum t_{i} \xi^{i}}\right) \bigwedge_{\alpha} \phi_{, \alpha}
$$

Since $C_{\sum t_{i} \xi^{i}(u)}$ is nilpotent for any small $u$ and any $t$, the preceding determinant is never zero. Hence $F$ is an immersion of entire $B_{\epsilon}^{r} \times \mathbf{C}^{n-r}$. It follows that $V$ is a subset of a strip of developable submanifold which has closed Gauss leaves.

Remark. In the real case, that is, if $f$ is an isometric immersion into $\mathbf{R}^{N}$ with relative nullity $\nu_{f}>0$, the proof of Proposition 1 implies that, $f$ is Gauss complete if and only if the conullity operators do not have any nonzero real eigenvalues. 


\section{Conullity foliation.}

Let us consider the ruling Gauss map

$$
\Phi: M^{\prime} \rightarrow G r_{\mathbf{C}}(n-r, N),
$$

defined by $\Phi(x)=d \iota\left(\mathcal{L}_{x}\right)$, which is identified with the $(n-r)$-dimensional complex vector subspace of $\mathbf{C}^{N}$ parallel to $d \iota\left(\mathcal{L}_{x}\right)$. If $M^{\prime}$ is a cylinder (with respect to the Gauss foliation $\mathcal{L}$ ), then all the leaves of $\mathcal{L}$ are parallel and $\Phi$ is constant. The nonzero rank of $\Phi$ is thus a measure of the failure of $M^{\prime}$ to be a cylinder. Let $M^{\prime \prime} \subseteq M^{\prime}$ be the open dense subset where $\Phi$ reaches its maximum rank. Then within $M^{\prime \prime}$, the kernel distribution $\operatorname{ker}\left(d \Phi_{x}\right)$ is of constant rank, and it defines a foliation $\mathcal{K}$. Note that $\mathcal{L} \subseteq \mathcal{K} \subseteq T M$, and $\mathcal{K}=T M$ iff $M$ is itself a cylinder with respect to the Gauss foliation $\mathcal{L}$. This foliation $\mathcal{K}$ was first studied by Vitter in [V], and we will follow him and call $\mathcal{K}$ the conullity foliation of $M$ (strictly speaking, of $M^{\prime \prime}$ ). From the definition, it is easy to see that if $x \in M^{\prime \prime}$, then the entire Gauss leaf $\mathcal{L}_{x} \subseteq M^{\prime \prime}$. (Note that by $[\mathrm{F}]$, when $M$ is complete, the Gauss leaf $\mathcal{L}_{y}$ for any $y \in M^{\prime}$ is closed in $M$, and thus $\left.\iota\right|_{\mathcal{L}_{y}}$ is a biholomorphism onto a linear subvariety $\mathbf{C}^{n-r}$ ).

The proof of the following lemma is omitted since it is a straight-forward computation.

Lemma 1. For any $x \in M^{\prime \prime}, \mathcal{K}_{x}=\mathcal{L}_{x} \oplus\left\{\bigcap_{X} \operatorname{ker}\left(C_{X}\right)\right\}$, where the intersection is taken over all $X$ in $\mathcal{L}_{x}$.

So each leaf of $\mathcal{K}$ is a cylinder consisting of parallel leaves of $\mathcal{L}$. If $\operatorname{rank} \mathcal{K} \equiv \operatorname{dim} \mathcal{K}_{x}$ (for any $x$ ) is bigger than $\operatorname{rank} \mathcal{L} \equiv \operatorname{dim} \mathcal{L}_{x}$, then although $M^{\prime \prime}$ may not be a cylinder, it is foliated by $\mathcal{L}$-cylinders. Vitter called such $M^{\prime \prime}$ "twisted cylinders". Vitter raised in [V] the question (cf. the Introduction) of when will rank $\mathcal{K}$ be greater than rank $\mathcal{L}$ ? The following theorem says that when the Gauss rank $r$ is less than 5 or equal to $n-1$, this will be the case. We also produce examples in $\S 3$ showing that for $5 \leq r \leq n-2$, there are complete developable hypersurfaces $M^{n} \subseteq \mathbf{C}^{n+1}$ with Gauss rank $r$ but with $\mathcal{K}=\mathcal{L}$. This is the other extreme of $\mathcal{K}=T M$, showing that, in general, complete developable complex submanifolds need not be twisted cylinders.

Theorem 1. Suppose an immersed complex submanifold $\left(M^{n}, \iota\right)$ in $\mathbf{C}^{N}$ is complete and has Gauss rank $r<n$. If either $r=n-1$ or $r \leq 4$, then the conullity foliation $\mathcal{K}$ of $M$ is strictly bigger than $\mathcal{L}$ in ranks. 
Remark 1. The case $r=2$ is due to Vitter ([V]) and Dajczer-Rodriquez ([D-R]). In fact, the result in [D-R] is much stronger: its Theorem 2 says that, for any minimal immersion $f: M^{2 n} \rightarrow \mathbf{R}^{N}$ of a complete Kähler manifold $M^{2 n}$, if the real Gauss rank (namely, the real dimension of the image of the Gauss map) $r \leq 4$, then either it is a cylinder: $M^{2 n}=N^{4} \times$ $\mathbf{R}^{2 n-4}$ and $f=f_{1} \times$ id, or $M^{2 n}$ admits a complex foliation whose leaves are isometrically mapped by $f$ onto affine subspaces $\mathbf{R}^{2 n-2}$.

Proof. Fix a generic point $x \in M^{\prime \prime}$. Let $\left\{X_{i} ; Y_{\alpha} ; Z_{\mu}\right\}$ be an unitary basis of $\mathbf{C}^{N}$ at $x$, such that $T_{x} \mathcal{L}_{x}$ is spanned by the $X_{i}$ 's, and the $Z_{\mu}$ 's are perpendicular to $T_{x} M$. Here again we use the index range

$$
1 \leq \alpha, \beta, \ldots \leq r, \quad r+1 \leq i, j, \ldots \leq n, \quad n+1 \leq \mu, \nu, \ldots \leq N
$$

Extend this basis into a tangent frame $\left\{\widetilde{X}_{i} ; \widetilde{Y}_{\alpha} ; \widetilde{Z}_{\mu}\right\}$ in a neighborhood of $x$ so that they are parallel along the leaves of $\mathcal{L}$. The developability of the foliation $\mathcal{L}$ guarantees that this can be done.

For any $i$ or $\mu$, consider the complex $r \times r$ matrices $A^{i}, E^{\mu}$ defined by

$$
A_{\alpha \beta}^{i}=-\left\langle\nabla_{Y_{\beta}} \tilde{X}_{i}, \bar{Y}_{\alpha}\right\rangle, \quad E_{\alpha \beta}^{\mu}=\left\langle\nabla_{Y_{\beta}} \tilde{Y}_{\alpha}, \bar{Z}_{\mu}\right\rangle
$$

$A^{i}$ is just the matrix of the conullity operator $C_{X_{i}}$ with respect to the basis $\left\{Y_{\alpha}\right\}$, while $E^{\mu}$ is just the $Z_{\mu}$-component of the second fundamental form $\mathrm{II}\left(Y_{\alpha}, Y_{\beta}\right)=\sum_{\mu} E_{\alpha \beta}^{\mu} Z_{\mu}$. We claim:

(1) $E^{\mu}$ and $E^{\mu} A^{i}$ are symmetric for any $i$, $\mu$, and $\sum_{\mu=n+1}^{N} E^{\mu} \overline{E^{\mu}}>0$;

(2) For any $t=\left(t_{r+1}, \ldots, t_{n}\right) \in \mathbf{C}^{n-r}, \sum_{i=r+1}^{n} t_{i} A^{i}$ is nilpotent.

The symmetry of $E^{\mu}$ and $E^{\mu} A^{i}$ is a consequence of the symmetry of the second fundamental form II itself and the symmetry of $C_{X}$ with respect to II. The meaning of $\sum_{\mu} E^{\mu} \overline{E^{\mu}}>0$ in (1) is that the matrix $\sum_{\mu} E^{\mu} \overline{E^{\mu}}$ is Hermitian positive definite. The fact that it is Hermitian positive semidefinite is clear. Suppose it is degenerate, then there is a nonzero column vector $v=\left(v_{1} \ldots v_{r}\right)^{t}$ so that $\sum_{\mu} E^{\mu} \bar{E}^{\mu} v=0$. This implies $\sum_{\mu} E^{\mu} \bar{v}=0$, so that if $Y=\sum_{\beta} \bar{v}_{\beta} Y_{\beta}$, then $\left\langle\nabla_{Y} \widetilde{Y}_{\alpha}, \bar{Z}_{\mu}\right\rangle=0$ for every $\alpha$. Thus $\operatorname{II}\left(Y, Y_{\alpha}\right)=0$ for every $\alpha$, implying that the second fundamental form II is degenerate on $\mathcal{L}^{\perp}$, contradiction. This proves (1), and (2) is just the Abe nilpotency theorem. 
Let $N_{i}=\left\{v \in \mathbf{C}^{r} \mid A^{i} v=0\right\}$. Here $v$ is a column vector. Then $N_{i}$ is just the kernel of $C_{X_{i}}$. So by Lemma 1 above, we know that the proof of Theorem 1 will be complete as soon as we prove the following algebraic result, Proposition 2. (In the case $r=n-1$, there is only one $A^{i}$, so the nilpotency always guarantees that the (common) kernel is non-trivial).

Proposition 2. Let $\left\{A^{i}\right\}$ be linear transformations of $\mathbf{C}^{r}$ and let $\left\{E^{\mu}\right\}$ be symmetric bilinear forms on $\mathbf{C}^{r}$ so that each $A^{i}$ is symmetric with respect to all the $E^{\mu}$ 's. With respect to a basis $\left\{e_{1}, \ldots, e_{r}\right\}$ of $\mathbf{C}^{r}$, let the matrices of $A^{i}$ and $E^{\mu}$ continue to be denoted by $A^{i}$ and $E^{\mu}$ respectively for the sake of simplicity. Suppose $A^{i}$ and $E^{\mu}$ satisfy conditions (1) and (2) above. If $r \leq 4$, then $\cap_{i} N_{i} \neq 0$.

\section{Remark.}

(a) For linear transformations $\left\{A^{i}\right\}$ and bilinear forms $\left\{E^{\mu}\right\}$, if their matrices satisfy (1) and (2) with respect to one basis of $\mathbf{C}^{r}$, they do so with respect to all bases.

(b) The symmetry of the bilinear forms $E^{\mu}$ and the symmetry of each $A^{i}$ with respect to $E^{\mu}$ of course imply that the matrices $E^{\mu}$ and $E^{\mu} A^{i}$ are symmetric. So the first part of condition (1) is automatically satisfied.

The rest of the section will be devoted to the proof of the this algebraic proposition, which is the main technical part of this article.

Proof of Proposition 2. Let $\mathcal{V}=\operatorname{span}\left\{A^{r+1}, \ldots, A^{n}\right\}$. Notice that any matrix $A \in \mathcal{V}$ also satisfies conditions (1) and (2) above. Let $l$ be the maximum rank of all the $A \in \mathcal{V}$. We claim that there is a basis $\left\{B^{1}, \ldots, B^{s}\right\}$ of $\mathcal{V}$ so that all the $B^{j}$ 's have the same Jordan canonical form and the same rank $l$. This can be seen as follows. Let $\mathcal{V}^{0}$ be the subset of $\mathcal{V}$ consisting of all the elements of $\mathcal{V}$ which have rank $l$. Then $\mathcal{V}^{0}$ is an open dense subset of $\mathcal{V}$. Each $A \in \mathcal{V}^{0}$ is a nilpotent $r \times r$ matrix of rank $l$, so the number of possibilities of the Jordan normal form of such an $A$ is finite in number. Thus $\mathcal{V}^{0}$ is partitioned into a finite number of equivalence classes $\mathcal{V}_{1}, \ldots, \mathcal{V}_{p}$, where the elements in each $\mathcal{V}_{\sigma}$ have the same Jordan normal form and if $\sigma \neq \rho$, the Jordan normal forms of $\mathcal{V}_{\sigma}$ and $\mathcal{V}_{\rho}$ are distinct. Suppose none of $\mathcal{V}_{1}, \ldots, \mathcal{V}_{p}$ contains a basis, then each of $\operatorname{span} \mathcal{V}_{1}, \ldots, \operatorname{span} \mathcal{V}_{p}$ is contained in a hyperplane, and $\mathcal{V}^{0}=\mathcal{V}_{1} \cup \ldots \cup \mathcal{V}_{p} \subseteq \operatorname{span} \mathcal{V}_{1} \cup \ldots \cup \operatorname{span} \mathcal{V}_{p}=$ a finite number of hyperplanes. Contradiction. Thus one of them, say $\mathcal{V}_{1}$, contains a basis $B^{1}, \ldots, B^{s}$ of $\mathcal{V}$, and this is the desired basis. 
For simplicity, we shall henceforth assume that the given $A^{r+1}, \ldots, A^{n}$ are such a basis of $\mathcal{V}=\operatorname{span}\left\{A^{r+1}, \ldots, A^{n}\right\}$, i.e., they are all of the same maximum rank $l(l<r)$, and all have the same Jordan normal form.

We will always consider the matrices $A^{i}$ as linear transformations on column vectors. Notice that for any nilpotent matrix $A, N(A) \cap R(A) \neq 0$, where $N(A)$ is its kernel, and $R(A)$ is its image. Another observation is the following:

$$
\text { For } r+1 \leq i, j \leq n, A^{j}\left(N\left(A^{i}\right)\right) \subseteq R\left(A^{i}\right) .
$$

Recall that $A^{i}$ and $A^{j}$ are all assumed to have maximum rank among all matrices in $\mathcal{V}$, so assertion $\left({ }^{*}\right)$ follows from a more general assertion: if $A, B$ are linear transformations on a vector space $V$ such that $\operatorname{rank} B \geq$ $\operatorname{rank}(t A+B)$ for all $t \in \mathbf{C}$, then $A(N(B)) \subseteq R(B)$. Indeed, if $R(B)=V$, this is trivial. So suppose $\operatorname{dim} R(B)=r<\operatorname{dim} V$. Then $\operatorname{dim} R(t A+B)<$ $\operatorname{dim} V$ for all $t$. If $A(N(B)) \nsubseteq R(B)$, then for some $v \in N(B), A(v) \notin R(B)$. However, by assumption, $\{R(t A+B) \mid t \in \mathbf{C}\}$ is a continuous family of proper subspaces of $V$ of which $R(B)$ is a member. Thus for a $t$ sufficiently small and $t \neq 0, A(v) \notin R(t A+B)$. But $A(v)=(t A+B)\left(\frac{1}{t} v\right)$, so we have $(t A+B)\left(\frac{1}{t} v\right) \notin R(t A+B)$, which is absurd. Assertion $\left(^{*}\right)$ is therefore proved.

We now divide our discussion into the following cases.

Case 1: $l=1$ (arbitrary $r)$.

Let $e_{1}, \ldots, e_{r}$ be a basis of $V=\mathbf{C}^{r}$ such that $R\left(A^{n}\right)=\mathbf{C} e_{1}, N\left(A^{n}\right)=$ $\operatorname{span}\left\{e_{1}, \ldots, e_{r-1}\right\}$. For any $r+1 \leq j \leq n-1$, by $\left({ }^{*}\right)$, we have $A^{j}\left(N\left(A^{n}\right)\right) \subseteq$ $R\left(A^{n}\right)$. So either $A^{j}\left(N\left(A^{n}\right)\right)=0$, in which case $N\left(A^{j}\right)=N\left(A^{n}\right)$, or $A^{j}\left(N\left(A^{n}\right)\right)=R\left(A^{n}\right)$, in which case $R\left(A^{j}\right)=R\left(A^{n}\right)$. Thus with respect to such a basis $e \equiv\left\{e_{1}, \ldots, e_{r-1}\right\}$, the condition of $l=1$ will force the transformations in $\mathcal{V}=\operatorname{span}\left\{A^{r+1}, \ldots, A^{n}\right\}$ to be, respectively, all in the form $B$ or all in the form $C$, where

$$
B=\left[\begin{array}{cccc}
0 & \ldots & 0 & * \\
\vdots & \ddots & \vdots & \vdots \\
0 & \ldots & 0 & * \\
0 & \ldots & 0 & 0
\end{array}\right] \text { and } C=\left[\begin{array}{cccc}
0 & * & \ldots & * \\
0 & 0 & \ldots & 0 \\
\vdots & \vdots & \ddots & \vdots \\
0 & 0 & \ldots & 0
\end{array}\right]
$$

In particular, $e_{1}$ is in $\bigcap N\left(A^{i}\right)$. 


\section{Remark.}

(a) If $r=2$, then $l \leq 1$. Thus Case 1 already proves Proposition 2 in case $r=2$.

(b) As noted above, the assumption $r \leq 4$ never entered into the preceding argument. Moreover, since $R\left(A^{j}\right) \subseteq N\left(A^{i}\right)$ for all $i, j$, we have $\sum_{j} R\left(A^{j}\right)=\bigcap_{i} N\left(A^{i}\right)$. These remarks will be important in the proof of Theorem 2 in $\S 4$.

Case 2: $\quad l=r-1, r=3,4$.

In this case, the Jordan form of any $A^{i}$ is $B=\left[\begin{array}{lll}0 & 0 & 0 \\ 1 & 0 & 0 \\ 0 & 1 & 0\end{array}\right]$ for $r=3$, and $=\left[\begin{array}{cccc}0 & 0 & 0 & 0 \\ 1 & 0 & 0 & 0 \\ 0 & 1 & 0 & 0 \\ 0 & 0 & 1 & 0\end{array}\right]$ for $r=4$. Let us assume that $A^{n}=B$. Now for each $\mu, E^{\mu}$ is a symmetric matrix. But $E^{\mu} B\left(\equiv E^{\mu} A^{n}\right)$ is also symmetric, so by multiplying out $E^{\mu} B$, we see that each $E^{\mu}$ has the form

$$
\left[\begin{array}{ccc}
a_{1} & a_{2} & a_{3} \\
a_{2} & a_{3} & 0 \\
a_{3} & 0 & 0
\end{array}\right] \text { or }\left[\begin{array}{cccc}
a_{1} & a_{2} & a_{3} & a_{4} \\
a_{2} & a_{3} & a_{4} & 0 \\
a_{3} & a_{4} & 0 & 0 \\
a_{4} & 0 & 0 & 0
\end{array}\right]
$$

depending on $r=3$ or $r=4$, respectively, where the $a_{j}$ 's are arbitrary complex numbers. We express this fact symbolically in the following way. Let $J=\left[\begin{array}{lll}0 & 0 & 1 \\ 0 & 1 & 0 \\ 1 & 0 & 0\end{array}\right]$ for $r=3$, and $=\left[\begin{array}{llll}0 & 0 & 0 & 1 \\ 0 & 0 & 1 & 0 \\ 0 & 1 & 0 & 0 \\ 1 & 0 & 0 & 0\end{array}\right]$ for $r=4$. Then

$$
E^{\mu}=J\left(a_{r} I+a_{r-1} B+\ldots+a_{1} B^{r-1}\right) .
$$

For at least one $E^{\mu}$, its $a_{r}$ must be non-zero, i.e., $\operatorname{det} E^{\mu} \neq 0$, since otherwise $E^{\mu} e_{r}=0$ for all $\mu$ and this would contradict condition (1). Take such an $E^{\mu}$, and denote it by $E$. Then $E=J f(B)$, where $f(s)=a_{r}+a_{r-1} s+\ldots+a_{1} s^{r-1}$ satisfies $f(0) \neq 0$. Let $g(s)$ be a complex analytic function near $s=0$ such that $g(s)^{2} f(s)=1$, and consider the change of basis in $\mathbf{C}^{r}$ under $g(B)^{t}$. With 
respect to this new basis, $A^{i}$ becomes $g(B)^{-1} A^{i} g(B)$ so that $A^{n}$ becomes $g(B)^{-1} A^{n} g(B)=g(B)^{-1} B g(B)=B$, while the bilinear form $E$ becomes

$$
\begin{aligned}
g(B)^{t} E g(B) & =g(B)^{t} J f(B) g(B) \\
& \left.=J g(B) f(B) g(B) \quad \text { (because } B^{t} J=J B\right) \\
& =J g(B)^{2} f(B)=J,
\end{aligned}
$$

because $g(s)^{2} f(s)=1$. Thus, we may assume, with respect to an appropriate basis of $\mathbf{C}^{r}$, that $E=J$ while $A^{n}=B$.

Relative to this new basis, since $J A^{i}$ is symmetric for each $i$, all $A^{i}$ are symmetric with respect to the anti-diagonal line.

Subcase 1: $l=2, r=3$.

For any $r+1 \leq j \leq n-1$, since $A^{j}$ is also trace-less, it can be written as

$$
A^{j}=\left[\begin{array}{ccc}
a & d & e \\
b & -2 a & d \\
c & b & a
\end{array}\right]
$$

By condition (2), $t B+A^{j}$ is nilpotent for any complex number $t$. So by a direct computation (e.g., expand $\left(t B+A^{j}\right)^{3}$ as a polynomial in $t$ and equate all the coefficients to 0 ), we get $a=d=e=0$. Thus any $A^{j}$ must be strictly lower triangular. In particular, $e_{3}$ is in the intersection of the kernels of all the $A^{j}$ 's.

Subcase 2: $l=3, r=4$.

Just as in the preceding subcase, we may write any $A^{j}(r+1 \leq j \leq n-1)$ as

$$
A^{j}=\left[\begin{array}{cccc}
a & b & c & d \\
f & -a & e & c \\
g & h & -a & b \\
k & g & f & a
\end{array}\right] .
$$

Again, by the condition that $t B+A^{j}$ is nilpotent for any $t$, we get after a computation:

$$
a=b=c=d=e=0 .
$$

So each $A^{j}$ is strictly lower triangular and $\bigcap_{j} N\left(A^{j}\right) \supseteq\left\{e_{4}\right\} \neq 0$.

Case 3: $r=4, l=2$. 
We divide the discussion into two subcases according to whether the Jordan normal form of the $A^{j}$ 's is

$$
C_{1}=\left[\begin{array}{llll}
0 & 0 & 0 & 0 \\
1 & 0 & 0 & 0 \\
0 & 0 & 0 & 0 \\
0 & 0 & 1 & 0
\end{array}\right] \quad \text { or } C_{2}=\left[\begin{array}{llll}
0 & 0 & 0 & 0 \\
1 & 0 & 0 & 0 \\
0 & 1 & 0 & 0 \\
0 & 0 & 0 & 0
\end{array}\right]
$$

Subcase 1: The Jordan form is $C_{1}$.

This is exactly the case when all $\left(A^{j}\right)^{2}=0$. So $N\left(A^{j}\right)=R\left(A^{j}\right)$. Let $\left\{e_{1}, e_{2}\right\}$ be a basis of $N\left(A^{n}\right)=R\left(A^{n}\right)$ and complete it to a basis $\left\{e_{1}, \ldots e_{4}\right\}$ of $\mathbf{C}^{4}$. Fix any $j$ between $r+1$ and $n-1$. Since $A^{j}\left(N\left(A^{n}\right)\right) \subseteq R\left(A^{n}\right)$ relative to $\left\{e_{1}, \ldots, e_{4}\right\}, A^{n}$ and $A^{j}$ are in the block form:

$$
A^{n}=\left[\begin{array}{ll}
0 & * \\
0 & 0
\end{array}\right] \text { and } A^{j}=\left[\begin{array}{ll}
* & * \\
0 & *
\end{array}\right] \text {. }
$$

By performing a change of bases within span $\left\{e_{1}, e_{2}\right\}$ and $\operatorname{span}\left\{e_{3}, e_{4}\right\}$ and making use of the nilpotence of $A^{n}$ and $A^{j}$, we may assume that

$$
A^{n}=\left[\begin{array}{cccc}
0 & 0 & a_{0} & b_{0} \\
0 & 0 & c_{0} & d_{0} \\
0 & 0 & 0 & 0 \\
0 & 0 & 0 & 0
\end{array}\right] \text { and } A^{j}=\left[\begin{array}{cccc}
0 & \alpha & a & b \\
0 & 0 & c & d \\
0 & 0 & 0 & \beta \\
0 & 0 & 0 & 0
\end{array}\right]
$$

If $\alpha \beta \neq 0$, then $R\left(A^{j}\right) \supseteq \operatorname{span}\left\{e_{1}, e_{3}\right\}$. Since $l=2$, necessarily $R\left(A^{j}\right)=$ $\operatorname{span}\left\{e_{1}, e_{3}\right\}$. Then $N\left(A^{j}\right)=R\left(A^{j}\right)=\operatorname{span}\left\{e_{1}, e_{3}\right\} \Longrightarrow a=c=0$. Repeat the same argument with $A^{j}$ replaced by $A^{j}+\epsilon A^{n}$ for a positive $\epsilon$, we conclude also that $a_{0}=c_{0}=0$. This contradicts the fact that $A^{n}$ has rank 2. So suppose $\beta=0$. We claim: $\alpha=0$. This is because $\beta=0 \Longrightarrow R\left(A^{j}\right) \subseteq$ $\operatorname{span}\left\{e_{1}, e_{2}\right\}, \Longrightarrow R\left(A^{j}\right)=\operatorname{span}\left\{e_{1}, e_{2}\right\}$ since $A^{j}$ has rank 2 . So if $\alpha \neq 0$, then $e_{2} \notin N\left(A^{j}\right)$, which contradicts $R\left(A^{j}\right)=N\left(A^{j}\right)$. The claim is proved. Similarly, if $\alpha=0$, then $\beta=0$. Therefore $N\left(A^{j}\right)=\operatorname{span}\left\{e_{1}, e_{2}\right\}=N\left(A^{n}\right)$ for all $j$.

Subcase 2: The Jordan form is $C_{2}$.

In this case, we have $N\left(A^{i}\right) \neq R\left(A^{i}\right)$ for any $i$.

Claim 1. For any $i, j, N\left(A^{i}\right) \cap N\left(A^{j}\right) \neq 0$.

Assume the contrary. Then we can find a basis $\left\{e_{1}, \ldots, e_{4}\right\}$ of $\mathbf{C}^{4}$ so that $N\left(A^{i}\right)=\operatorname{span}\left\{e_{1}, e_{2}\right\}$ and $N\left(A^{j}\right)=\operatorname{span}\left\{e_{3}, e_{4}\right\}$. Since $N\left(A^{i}\right) \neq$ 
$R\left(A^{i}\right)$, and $N\left(A^{i}\right) \cap R\left(A^{i}\right) \neq 0, \quad \operatorname{dim}\left(N\left(A^{i}\right) \cap R\left(A^{i}\right)\right)=1$. Thus by a change of bases within $\operatorname{span}\left\{e_{1}, e_{2}\right\}$ and $\operatorname{span}\left\{e_{3}, e_{4}\right\}$, we may assume that $R\left(A^{i}\right) \cap N\left(A^{i}\right)=\mathbf{C} e_{2}$, so that $A^{i}$ can be brought to the form:

$$
A^{i}=\left[\begin{array}{llll}
0 & 0 & 0 & a \\
0 & 0 & 1 & 0 \\
0 & 0 & 0 & 1 \\
0 & 0 & 0 & 0
\end{array}\right] .
$$

Because $A^{j}\left(N\left(A^{i}\right)\right) \subseteq R\left(A^{i}\right)=\left\{e_{2}, a e_{1}+e_{3}\right\}$, it follows that with respect to this new basis,

$$
A^{j}=\left[\begin{array}{cccc}
a c & a e & 0 & 0 \\
b & d & 0 & 0 \\
c & e & 0 & 0 \\
0 & 0 & 0 & 0
\end{array}\right] .
$$

Since $A^{j}$ is nilpotent and has rank equal to 2 , we have

$$
a c+d=0, \quad a(c d-b e)=0, \quad b e-c d \neq 0 .
$$

So $a=0=d, b e \neq 0$. Now look at the combination $t A^{i}+A^{j}$

$$
t A^{i}+A^{j}=\left[\begin{array}{cccc}
0 & 0 & 0 & 0 \\
b & 0 & t & 0 \\
c & e & 0 & t \\
0 & 0 & 0 & 0
\end{array}\right]
$$

Thus trace $\left(t A^{i}+A^{j}\right)^{2}=0$ implies $2 e t=0$. This being true for all $t, e=0$, and $A^{j}$ has rank 1. Contradiction. This completes the proof of Claim 1.

Claim 2. $\bigcap_{i=r+1}^{n} N\left(A^{i}\right) \neq 0$.

Assume the contrary. Since all $A^{i}$ have rank equal to 2, Claim 1 implies that there will be three matrices $A=A^{i}, B=A^{j}$ and $C=A^{k}$ such that $N(A) \cap N(B) \cap N(C)=0$, and a basis $\left\{e_{1}, \ldots, e_{4}\right\}$ such that

$$
N(A)=\operatorname{span}\left\{e_{1}, e_{2}\right\}, N(B)=\operatorname{span}\left\{e_{2}, e_{3}\right\}, N(C)=\operatorname{span}\left\{e_{3}, e_{1}\right\} .
$$

Let $V=\operatorname{span}\left\{e_{1}, e_{2}, e_{3}\right\}=N(A)+N(B)=N(B)+N(C)=N(C)+N(A)$ and $W=R(A) \cap R(B) \cap R(C)$. Since $e_{3} \in N(B) \cap N(C), A e_{3} \in R(B) \cap$ $R(C) \subseteq W$. Similarly, $B e_{1}, C e_{2}$ are also in $W$. Note that $A e_{3}, B e_{1}$, and $C e_{2}$ are all nonzero because $A, B$, and $C$ have rank 2 . Hence $\operatorname{dim} W \geq 1$. 
If $\operatorname{dim}(W)=2$, necessarily $R(A)=R(B)=R(C)$. Then with respect to a new basis $\left\{e_{1}^{\prime}, \ldots, e_{4}^{\prime}\right\}$ such that $R(A)=\operatorname{span}\left\{e_{1}^{\prime}, e_{2}^{\prime}\right\}$, we have

$$
A=\left[\begin{array}{cc}
A^{\prime} & * \\
0 & 0
\end{array}\right], B=\left[\begin{array}{cc}
B^{\prime} & * \\
0 & 0
\end{array}\right] \text { and } C=\left[\begin{array}{cc}
C^{\prime} & * \\
0 & 0
\end{array}\right]
$$

where $A^{\prime}, B^{\prime}$ and $C^{\prime}$ are $2 \times 2$ matrices and all their linear combinations are necessarily nilpotent. It is then easy to see that they must have a common kernel, which contradicts our assumption that $N(A) \cap N(B) \cap N(C)=0$. So $W$ must be one dimensional. Rescale $e_{i}$ if necessary, we may assume

$$
A e_{3}=B e_{1}=C e_{2}, \text { and it spans } W \text {. }
$$

(i) If $W \subseteq V$, let $A e_{3}=a e_{1}+b e_{2}+c e_{3}$, then since

$$
A+t B+s C=\left[\begin{array}{cccc}
t a & s a & a & * \\
t b & s b & b & * \\
t c & s c & c & * \\
0 & 0 & 0 & *
\end{array}\right]
$$

and is nilpotent for all $t, s$, its upper left $3 \times 3$ block is also nilpotent, hence trace-less. This implies that $a=b=c=0$, a contradiction.

(ii) $W$ is not contained in $V$. Replace $e_{4}$ by $A e_{3}$, then relative to the new basis,

$$
A=\left[\begin{array}{cccc}
0 & 0 & 0 & \alpha \\
0 & 0 & 0 & \gamma \\
0 & 0 & 0 & \alpha^{\prime} \\
0 & 0 & 1 & 0
\end{array}\right] ; \quad B=\left[\begin{array}{cccc}
0 & 0 & 0 & \delta^{\prime} \\
0 & 0 & 0 & \delta \\
0 & 0 & 0 & \epsilon \\
1 & 0 & 0 & 0
\end{array}\right] ; \quad C=\left[\begin{array}{cccc}
0 & 0 & 0 & \beta \\
0 & 0 & 0 & \beta^{\prime} \\
0 & 0 & 0 & \zeta \\
0 & 1 & 0 & 0
\end{array}\right] .
$$

Because $A^{2}, B^{2}$, and $C^{2}$ are trace-less, $\alpha^{\prime}=\delta^{\prime}=\beta^{\prime}=0$. Moreover, $A+$ $t B+s C$ is nilpotent for any $t$ or $s$, so $(A+t B+s C)^{2}$ is trace-less for any $t$ or $s$, and hence $\epsilon=-\alpha, \zeta=-\gamma$, and $\delta=-\beta$. Hence

$$
A+t B+s C=\left[\begin{array}{cccc}
0 & 0 & 0 & \alpha+s \beta \\
0 & 0 & 0 & \gamma-t \beta \\
0 & 0 & 0 & -t \alpha-s \gamma \\
t & s & 1 & 0
\end{array}\right]
$$

Now write $E^{\mu}$ with respect to the same basis as

$$
E^{\mu}=\left[\begin{array}{llll}
a & e & h & j \\
e & b & f & i \\
h & f & c & g \\
j & i & g & d
\end{array}\right]
$$


Then by the symmetry of $E^{\mu}(A+t B+s C)$ for any $t$ or $s$, we get $g=i=$ $j=0$ and

$$
d=-e \beta-h \alpha=e \beta-f \gamma=h \alpha+f \gamma .
$$

Sum up these three distinct expressions of $d$ gives $3 d=0$, so $E^{\mu} e_{4}=0$ for any $\mu$. This contradicts the fact that

$$
\sum_{\mu=n+1}^{N} E^{\mu} \overline{E^{\mu}}>0 .
$$

So we have completed the proof of Claim 2 and hence also the proof of Proposition 2.

Arguing along this line further, we get the following, whose proof is is left to the readers.

Proposition 3. Let $A^{i}, E^{\mu}$ be as in Proposition 2. Then for $2 \leq r \leq 4$, there always exists a basis $\left\{e_{1}, \ldots, e_{r}\right\}$ such that the linear span $\mathcal{A}_{r}$ of the transformations $A^{i}$ (on column vectors) is contained in one of the following linear spaces:

$$
\begin{array}{cc}
\mathcal{A}_{2} \subseteq\left\{\left[\begin{array}{ll}
0 & * \\
0 & 0
\end{array}\right]\right\} & \\
\mathcal{A}_{3} \subseteq\left\{\left[\begin{array}{lll}
0 & 0 & * \\
0 & 0 & * \\
0 & 0 & 0
\end{array}\right]\right\} & \text { or }\left\{\left[\begin{array}{lll}
0 & x & * \\
0 & 0 & x \\
0 & 0 & 0
\end{array}\right]\right\} \\
\mathcal{A}_{4} \subset\left\{\left[\begin{array}{llll}
0 & 0 & 0 & * \\
0 & 0 & 0 & * \\
0 & 0 & 0 & * \\
0 & 0 & 0 & 0
\end{array}\right]\right\} & \text { or }\left\{\left[\begin{array}{llll}
0 & * & * & * \\
0 & 0 & * & * \\
0 & 0 & 0 & 0 \\
0 & 0 & 0 & 0
\end{array}\right]\right\} \\
\text { or }\left\{\left[\begin{array}{llll}
0 & 0 & * & * \\
0 & 0 & x & * \\
0 & 0 & 0 & x \\
0 & 0 & 0 & 0
\end{array}\right]\right\} & \text { or }\left\{\left[\begin{array}{llll}
0 & x & y & * \\
0 & 0 & * & y \\
0 & 0 & 0 & x \\
0 & 0 & 0 & 0
\end{array}\right]\right\} .
\end{array}
$$

\section{Examples.}

First of all, let us give an example which shows that in Proposition 2, the upper bound for the Gauss rank $r$ is necessary. Its verification is straightforward computation, so will be omitted. 
Example 1. Let $A, B, E$ be given respectively by

$$
\left[\begin{array}{lllll}
0 & 0 & 0 & 0 & 0 \\
1 & 0 & 0 & 0 & 0 \\
0 & 1 & 0 & 0 & 0 \\
0 & 0 & 1 & 0 & 0 \\
0 & 0 & 0 & 1 & 0
\end{array}\right],\left[\begin{array}{ccccc}
0 & 0 & 1 & 0 & 0 \\
0 & 0 & 0 & -2 & 0 \\
0 & 0 & 0 & 0 & 1 \\
0 & 0 & 0 & 0 & 0 \\
0 & 0 & 0 & 0 & 0
\end{array}\right],\left[\begin{array}{lllll}
0 & 0 & 0 & 0 & 1 \\
0 & 0 & 0 & 1 & 0 \\
0 & 0 & 1 & 0 & 0 \\
0 & 1 & 0 & 0 & 0 \\
1 & 0 & 0 & 0 & 0
\end{array}\right] .
$$

Then $E$ is symmetric and non-singular, $E A, E B$ are symmetric, and for any complex numbers $t$ and $s$, the combination $t A+s B$ is always nilpotent. So they satisfy condition (1) and (2) in $\S 2$. But clearly, $N(A) \cap N(B)=0$.

The following lemma says that for any given $E$ and $\left\{A^{i}\right\}$ satisfying conditions (1) and (2) of $\S 2$, there is a properly embedded developable hypersurface $M^{n} \subseteq \mathbf{C}^{n+1}$ such that its conullity operators are given by these $A^{i}$, s (along $\{t=0\}$ ). It then follows that this lemma, coupled with Example 1, give a counterexample to Theorem 1 in case $r>4$ and $r \neq n-1$.

Lemma 2. Suppose $E, A^{r+1}, \ldots, A^{n}$ are complex $r \times r$ constant matrices such that $E$ is symmetric and non-singular, each $E A^{i}$ is symmetric, and $\sum_{i=r+1}^{n} t_{i} A^{i}$ is nilpotent for any $t=\left(t_{r+1}, \ldots, t_{n}\right) \in \mathbf{C}^{n-r}$. Let $B=(I+$ $\left.\sum_{i=r+1}^{n} t_{i} A^{i}\right)^{-1} E^{-1}$; it is well defined for any $t$ by the nilpotency assumption. Then the smooth complete hypersurface $M^{n} \subseteq \mathbf{C}^{n+1}$ defined by

$$
M^{n}=\left\{(z, t, w) \in \mathbf{C}^{r} \times \mathbf{C}^{n-r} \times \mathbf{C} \mid w=\sum_{\alpha, \beta=1}^{r} B_{\alpha \beta} z_{\alpha} z_{\beta}\right\}
$$

has Gauss rank $r<n$, and, along $t=0$, its conullity operators are given by the $-A^{i}$ 's.

Proof. Let $\left\{e_{a}\right\}, 1 \leq a \leq n+1$ be the standard basis of $\mathbf{C}^{n+1}$. As before, we will use the index range $1 \leq \alpha, \beta, \ldots \leq r$ and $r+1 \leq i, j, \ldots \leq n$. Consider

$$
\begin{aligned}
\phi(u) & =\sum_{\alpha} u_{\alpha} e_{\alpha}+\sum_{\alpha, \beta}\left(E^{-1}\right)_{\alpha \beta} u_{\alpha} u_{\beta} e_{n+1} \\
\xi^{i}(u) & =\sum_{\alpha, \beta} A_{\alpha \beta}^{i} u_{\alpha} e_{\beta}+e_{i}+\left(A^{i} E^{-1}\right)_{\alpha \beta} u_{\alpha} u_{\beta} e_{n+1}
\end{aligned}
$$

where $u=\left(u_{1}, \ldots, u_{r}\right) \in \mathbf{C}^{r}$. Then $F(u, t)=\phi(u)+\sum t_{i} \xi^{i}(u)$ gives a global holomorphic parametrization of $M$ for the following reason. If 
we let $C=\left(I+\sum_{i} t_{i} A^{i}\right)$, then $B=C^{-1} E^{-1}$ is symmetric, $F(u, t)=$ $\left(u C, t, u C E^{-1} u^{t}\right)$, and

$$
\begin{aligned}
u C E^{-1} u^{t} & =u C\left(E^{-1}\right)^{t} u^{t}=u C(C B)^{t} u^{t} \\
& =(u C) B(u C)^{t}=\sum_{\alpha, \beta} B_{\alpha \beta}(u C)_{\alpha}(u C)_{\beta}
\end{aligned}
$$

Comparing with the definition of $M$, this shows that the image of $F$ is exactly $M$. Note that, by definition, $M$ is foliated by linear subvarieties of dimension $(n-r)$. Since $E^{-1}$ and $A^{i} E^{-1}$ are both symmetric (the symmetry of $A^{i} E^{-1}$ is straightforward to show), the partial derivatives with respect to $u_{\alpha}$ are

$$
\begin{aligned}
\phi_{, \alpha} & =e_{\alpha}+2 \sum_{\beta}\left(E^{-1}\right)_{\alpha \beta} u_{\beta} e_{n+1} \\
\xi_{, \alpha}^{i} & =\sum_{\beta} A_{\alpha \beta}^{i} e_{\beta}+2 \sum_{\beta}\left(A^{i} E^{-1}\right)_{\alpha \beta} u_{\beta} e_{n+1}
\end{aligned}
$$

So for any $i$ or $\alpha$, we have

$$
\xi_{, \alpha}^{i}=\sum_{\beta} A_{\alpha \beta}^{i} \phi_{, \beta} .
$$

Therefore along each linear subvariety $\mathcal{L}_{u}=F\left(\{u\} \times \mathbf{C}^{n-r}\right)$, the tangent space $T M$ is constantly spanned by $\phi_{, \alpha}$ and $\xi^{i}$, where $\alpha=1, \ldots, r$ and $i=r+1, \ldots, n$. That is, the ruling foliation $\mathcal{L}$ is a developable foliation. A direct computation of $\left\{\nabla_{\phi, \alpha} \phi_{, \beta} \mid\right.$ all $\left.\alpha, \beta\right\}$ (taking into account of $\left.\nabla_{\phi, \alpha} \phi_{, \beta}=\phi_{, \alpha \beta}\right)$ shows that the second fundamental form is nondegenerate on span $\left\{\phi_{, \alpha} \mid\right.$ all $\left.\alpha\right\}$ along $\{t=0\}=F\left(\mathbf{C}^{r} \times\{0\}\right)$, so that $\mathcal{L}$ is indeed the Gauss foliation of $M$. We claim that the conullity operator along $\mathbf{C}^{r} \times\{0\}$ are just the $\left\{-A^{i}\right\}$. Briefly, this is because at a point of $\mathbf{C}^{r} \times\{0\}$,

$$
\nabla_{\phi, \alpha} \xi^{i}=\xi_{, \alpha}^{i}=\sum_{\beta} A_{\alpha \beta}^{i} \phi_{, \beta},
$$

and $\nabla_{\xi^{j}} \xi^{i}=0$ for all $i, j$, so that if $Y_{\alpha}$ is the orthogonal projection of $\phi_{, \alpha}$ on $\mathcal{L}^{\perp}$ at that point, then

$$
\nabla_{Y_{\alpha}} \xi^{i}=\sum_{\beta} A_{\alpha \beta}^{i} \phi_{, \beta}
$$

From this, it follows immediately from the definition that

$$
C_{\xi^{i}}\left(Y_{\alpha}\right)=-\sum_{\beta} A_{\alpha \beta}^{i} Y_{\beta}
$$


Note that this construction also works for real developable submanifolds. For example, one may state the following

Lemma 3. Suppose $E, A^{r+1}, \ldots, A^{n}$ are $r \times r$ real matrices such that $E$ is symmetric and non-singular, each $E A^{i}$ is symmetric, and $\sum_{i=r+1}^{n} t_{i} A^{i}$ has no non-zero real eigenvalue for any $t=\left(t_{r+1}, \ldots, t_{n}\right) \in \mathbf{R}^{n-r}$. Let $B=\left(I+\sum_{i=r+1}^{n} t_{i} A^{i}\right)^{-1} E^{-1} ;$ it is well defined for any real $t$. Then the smooth complete hypersurface $M^{n} \subseteq \mathbf{R}^{n+1}$ defined by

$$
M^{n}=\left\{(z, t, w) \in \mathbf{R}^{r} \times \mathbf{R}^{n-r} \times \mathbf{R} \mid w=\sum_{\alpha, \beta=1}^{r} B_{\alpha \beta} z_{\alpha} z_{\beta}\right\}
$$

has Gauss rank $r<n$, and, along $t=0$, its conullity operators are given by the $-A^{i}$ 's.

In particular, the conullity operators for complete real analytic developable submanifolds in $\mathbf{R}^{N}$ are no longer always nilpotent.

Example 2. Consider

$$
E=\left[\begin{array}{cc}
1 & 0 \\
0 & -1
\end{array}\right], \quad A=\left[\begin{array}{cc}
0 & 1 \\
-1 & 0
\end{array}\right]
$$

Then both $E$ and $E A$ are symmetric, $E$ is non-singular, and $A$ has no real eigenvalue. The corresponding manifold is the complete smooth hypersurface $M^{3} \subseteq \mathbf{R}^{4}$ defined by

$$
M^{3}=\left\{(x, y, t, w) \in \mathbf{R}^{4} \mid w=\left(x^{2}-y^{2}+2 t x y\right) /\left(1+t^{2}\right)\right\}
$$

This cubic threefold has Gauss rank $r=2$, and it is not a cylinder. Note that the conullity operator is not nilpotent here.

Remark. For an immersed complex submanifold $\left(M^{n}, \iota\right)$ in $\mathbf{C}^{N}$, consider the projective ruling Gauss map

$$
\begin{aligned}
\Phi^{\prime}: M^{\prime} & \longrightarrow G=G r\left(\mathbf{C P}^{n-r}, \mathbf{C P}^{N}\right) \\
x & \longmapsto\left[\mathcal{L}_{x}\right] .
\end{aligned}
$$

Let $\Sigma_{M}$ be the image. It is an immersed complex submanifold of dimension $r$ in $G$. The examples in Lemma 2 are exactly those $M$ whose $\Sigma_{M}$ has vanishing third fundamental forms in $G$. This connection was brought to our attention by Robert Bryant. 


\section{The image distribution.}

Let us continue the discussion along the line of Theorem 1. Recall that the conullity foliation $\mathcal{K}$ on $M^{\prime \prime}$ is the kernel of the differential of the ruling Gauss map $\Phi$ defined by $\Phi(x)=d \iota\left(\mathcal{L}_{x}\right)$ and $\mathcal{K}_{x}=\mathcal{L}_{x} \oplus\left\{\bigcap_{X} \operatorname{ker}\left(C_{X}\right)\right\}$ (Lemma 1). Now consider for each $x \in M^{\prime \prime}$ the subspace

$$
\mathcal{R}_{x}=\mathcal{L}_{x} \oplus \sum_{X \in \mathcal{L}_{x}} R\left(C_{X}\right)
$$

where $R\left(C_{X}\right)$ is the range (image space) of the conullity operator $C_{X}$ at $x$. Let $M^{\prime \prime \prime}$ be the open dense subset of $M^{\prime \prime}$ where these subspaces $\mathcal{R}_{x}$ have constant (maximal) rank. Thus $\mathcal{R}$ is a holomorphic distribution in $M^{\prime \prime \prime}$ contained in $\mathcal{K}$. The following lemma is needed for the proof of Theorem 2.

Lemma 4. Suppose $M^{n} \subseteq \mathbf{C}^{N}$ is a piece of complex submanifold with Gauss rank $r$. For a point $x \in M^{\prime \prime \prime}$, let $U$ be a complex $r$-dimensional submanifold of $M$ containing $x$ and is transversal to $\mathcal{L}$, and let $u=\left(u_{1}, \ldots, u_{r}\right)$ be a holomorphic coordinate system on $U$. Suppose $\left\{\xi^{i}(u)\right\}$ is a holomorphic frame of $\mathcal{L}$ along $U$ constant along the leaves of $\mathcal{L}$ as usual. Then as a subbundle of $T_{x} M$,

$$
\mathcal{R}=\operatorname{span}\left\{\xi^{\mathrm{i}}, \xi_{, \alpha}^{\mathrm{j}} ; \quad 1 \leq \alpha \leq \mathrm{r}, \mathrm{r}<\mathrm{i}, \mathrm{j} \leq \mathrm{n}\right\},
$$

where as usual, $\xi_{, \alpha}^{j} \equiv \partial \xi^{j} / \partial u_{\alpha}$.

Proof. Suppose $U$ is parametrized by $\phi(u)$. Then $M$ is parametrized by $F(u, t)=\phi(u)+\sum t_{i} \xi^{i}(u)$, and $\left\{\xi^{j}, \phi_{, \alpha}\right\}$ are a basis of vector fields tangent to $M$ (shrink $U$ if necessary). The developability of $\mathcal{L}$ in terms of the second fundamental form implies that all the $\xi_{, \alpha}^{i}$ 's are tangent to $M$. Hence we may write

$$
\xi_{, \alpha}^{i}=\sum_{\beta} A_{\alpha \beta}^{i} \phi_{, \beta}+\sum_{j} B_{j}^{i} \xi^{j} .
$$

Also write $\phi_{, \beta}=X_{\beta}+\sum_{j} D_{\beta j} \xi^{j}$, where $X_{\beta}$ is the component of $\phi_{, \beta}$ orthogonal to $\mathcal{L}$. Then,

$$
\xi_{, \alpha}^{i}=\sum_{\beta} A_{\alpha \beta}^{i} X_{\beta}+\sum_{j}\left(\sum_{\beta} A_{\alpha \beta}^{i} D_{\beta j}+B_{j}^{i}\right) \xi^{j} .
$$


By the definition of the conullity operator, the following is valid at each point of $M$ :

$$
\begin{aligned}
C_{\xi^{i}}\left(\partial / \partial u_{\alpha}\right) & =-\left(\nabla_{\partial / \partial u_{\alpha}} \xi^{i}\right)^{\perp}=-\left(\xi_{, \alpha}^{i}\right)^{\perp} \\
& =-\sum_{\beta} A_{\alpha \beta}^{i} X_{\beta} \\
& =-\xi_{, \alpha}^{i}+\sum_{j}\left(\sum_{\beta} A_{\alpha \beta}^{i} D_{\beta j}+B_{j}^{i}\right) \xi^{j} .
\end{aligned}
$$

The asserted equality of the lemma now follows from the definition of $\mathcal{R}$.

Theorem 2. For a complete immersed complex submanifold $\left(M^{n}, \iota\right)$ in $\mathbf{C}^{N}$ with Gauss rank $r<n$, if $\mathcal{R} \subseteq \mathcal{K}$, then $\mathcal{R}$ is a ruling foliation, i.e., the (image under $\iota$ of the) leaves of $\mathcal{R}$ are open subsets of linear subvarieties in $\mathbf{C}^{N}$.

In particular, if the maximum rank $l$ of all $C_{X}$ is 1 , then $\mathcal{R} \subseteq \mathcal{K}$, so within $M^{\prime \prime \prime}$, the Gauss foliation $\mathcal{L}$ is contained in a ruling foliation $\mathcal{R}$, which has strictly larger rank when $M$ is not a cylinder.

Proof. When $l=1$, Case 1 in the proof of Proposition 2 in $\S 2$ (see the Remark after Case 1) says that $\mathcal{R}_{x} \subseteq \mathcal{K}_{x}$ for any point $x \in M^{\prime \prime \prime}$. So assuming the first part of Theorem 2, the second part follows.

To prove the first part, let us assume that $\mathcal{R} \neq \mathcal{L}$, as otherwise all $C_{X}=0$ and $M$ would be an $\mathcal{L}$-cylinder. Fix a point $x \in M^{\prime \prime \prime}$. Let $W$ be a neighborhood of $x$ in $M^{\prime \prime \prime}$ which is a union of the leaves of the foliation $\mathcal{K}$ near $x$. We shall prove that $\mathcal{R}$ is integrable in $W$ and that its integral submanifolds are subsets of linear subvarieties. Now $\mathcal{R} \subseteq \mathcal{K}$ by assumption, so if $\mathcal{R}$ is restricted to a leaf $K$ of $\mathcal{K}$ in $W, \mathcal{R}$ becomes a subbundle of the tangent bundle $T \mathcal{K}$ of $\mathcal{K}$. If $\mathcal{R}$ is integrable, its integral submanifolds must therefore foliate each leaf $K$ of $\mathcal{K}$. To prove the theorem, it therefore suffices to restrict $\mathcal{R}$ to a fixed leaf $K_{0}$ of $\mathcal{K}$. To this end, we proceed as follows.

Since $\mathcal{L}$ and $\mathcal{K}$ are foliations on $W$ and $\mathcal{L} \subseteq \mathcal{K}$ in a self-explanatory sense, there exists a holomorphic coordinate system

$$
\left(u^{\prime}, u^{\prime \prime}, v\right) \equiv\left(u_{1}^{\prime}, \ldots, u_{s}^{\prime}, u_{s+1}^{\prime \prime}, \ldots, u_{r}^{\prime \prime}, v_{r+1}, \ldots, v_{n}\right)
$$

on $W$, such that

the leaves of $\mathcal{K}$ are defined by $\left\{u^{\prime}=\right.$ constant $\}$, and

the leaves of $\mathcal{L}$ are defined by $\left\{u^{\prime}=\right.$ constant, $u^{\prime \prime}=$ constant $\}$. 
For any leaf $K$ of $\mathcal{K}$ in $W$, if $p$ and $q$ are points in $K$, then $\mathcal{L}_{p}$ and $\mathcal{L}_{q}$ are parallel linear subvarieties. This is because the leaves of $\mathcal{K}$ are the level sets of the Gauss ruling map $\Phi$. Consequently we may choose a holomorphic frame $\left\{\xi^{r}, \ldots, \xi^{n}\right\}$ of $\mathcal{L}$ in $W$ so that $\left\{\xi^{i}\right\}$ is independent of $u^{\prime \prime}$ and $v$. Thus each $\xi$ is a function of $u^{\prime}$ alone, i.e., $\xi^{i}=\xi^{i}\left(u^{\prime}\right)$. Now fix a leaf $K_{0}$ of $\mathcal{K}$. By Lemma 4 , we have in $K_{0}$,

$$
\mathcal{R}=\operatorname{span}\left\{\xi^{i}, \partial \xi^{j} / \partial u_{\sigma}^{\prime} \mid 1 \leq \sigma \leq s, r<i, j \leq n\right\} .
$$

Since each $\partial \xi^{j} / \partial u_{\sigma}^{\prime}$ is necessarily also independent of $u^{\prime \prime}$ and $v$, the distribution $\mathcal{R}$ in $K_{0}$ is independent of $u^{\prime \prime}$ and $v$. That is, $\mathcal{R}$ is a constant distribution (i.e., parallel with respect to $\mathbf{C}^{N}$ ) on $K_{0}$. In particular, it is a totally geodesic (auto-parallel) distribution on $K_{0}$. Because totally geodesic distributions with respect to a torsionless connection must be integrable, $\mathcal{R}$ is integrable on $K_{0}$. The constancy of $\mathcal{R}$ on $K_{0}$ then leads trivially to the fact that its integral manifolds are subsets of linear subvarieties.

The following special case is due to Vitter ([V]).

Corollary 1. For a complete immersed complex submanifold $\left(M^{n}, \iota\right)$ in $\mathbf{C}^{N}$ with Gauss rank $r=2$, if $M$ is not a cylinder, then $\mathcal{R}=\mathcal{K}$ is a ruling foliation with $n-1$ dimensional leaves.

Proof. In this case, $C_{X}$ are $2 \times 2$ nilpotent matrices which are not all zero, so at any point $x \in M^{\prime \prime \prime}, \mathcal{K}_{x}=\mathcal{R}_{x} \cong \mathbf{C}^{n-1}$.

Fix a point $x \in M^{\prime \prime \prime}$ and an unitary frame for $\mathcal{L}^{\perp}$ in a small neighborhood $U$ of $x$. Denote by $\mathcal{A}_{y}=\operatorname{span}\left\{C_{1}, \ldots, C_{k}\right\}$ the subspace of $M_{r \times r}(\mathbf{C})$ spanned by the conullity operators at $y \in U$. The condition $\mathcal{R} \subseteq \mathcal{K}$ can be equivalently stated as $C_{i} C_{j}=0$ in $U$, for any $i, j$. On the linear algebra level, since $\sum R\left(C_{i}\right) \subseteq \bigcap N\left(C_{j}\right)$, we can always choose a basis $\left\{e_{1}, \ldots, e_{r}\right\}$ of each $\mathcal{L}_{x}^{\perp}$ such that $\left\{e_{1}, \ldots, e_{p}\right\}$ spans $\sum R\left(C_{i}\right)$, while $\left\{e_{1}, \ldots, e_{q}\right\}$ spans $\bigcap N\left(C_{j}\right)$, where $0 \leq p \leq q \leq r$. So with respect to this basis, all the $C_{i}$ are in the following block form

$$
\left[\begin{array}{cc}
0_{p \times q} & * \\
0 & 0
\end{array}\right] .
$$

Conversely, for any $0 \leq p \leq q \leq r$, any two matrices in the above block form have zero product. Incidentally, this way of expressing the fact that $\mathcal{R} \subseteq \mathcal{K}$ 
gives a direct proof that if the maximum rank of each conullity operator is 1 then $\mathcal{R} \subseteq \mathcal{K}$.

Let us conclude this section by an example, which falls into the situation of $\mathcal{R} \subseteq \mathcal{K}$.

Example 3. Consider the quintic hypersurface

$$
M^{4}=\left\{z \in \mathbf{C}^{5} \mid F(z) \equiv z_{1}+z_{2}\left(z_{4}^{2}+z_{5}^{2}\right)+z_{3}\left(z_{4}^{2}+z_{5}^{2}\right)^{2}=0\right\} \subseteq \mathbf{C}^{5} .
$$

It is a graph, hence smooth and properly embedded. The Gauss rank is 3 , and $\mathcal{R}=\mathcal{K}$ is a $\mathbf{C}^{2}$ ruling.

This example can be easily generalized to the following.

Let $U^{a} \subseteq \mathbf{C}^{a}$ be an open subset, $V^{b}$ be a complex manifold of dimension $b$. Let $h: U \rightarrow V$ and $f: V \rightarrow \mathbf{C}^{m} \backslash\{0\}$ be holomorphic maps. Consider the complex hypersurface

$$
M^{a+m-1}=\left\{(u, z) \in U \times \mathbf{C}^{m} \mid f(h(u)) \cdot z=0\right\} \subseteq \mathbf{C}^{a+m}
$$

where $w \cdot z=w_{1} z_{1}+\ldots+w_{m} z_{m}$. Suppose $h$ has a $b$-dimensional image and $f$ is non-degenerate in the sense that $f \wedge f_{v_{1}} \wedge \ldots \wedge f_{v_{b}}$ is not identically zero. Here subscripts denote partial differentiation with respect to a local coordinate system in $V$. We then have

$$
\mathcal{K} \supseteq \mathcal{R}=\{\bar{f}\}^{\perp} \supseteq \mathcal{L}=\left\{\bar{f}, \overline{f_{v_{1}}}, \ldots, \overline{f_{v_{b}}}\right\}^{\perp} .
$$

Here $\perp$ means the orthogonal complement in $\mathbf{C}^{m}$. Of course $M$ could be closed in $\mathbf{C}^{a+m}$ for suitably chosen data.

In Example 3, $h\left(z_{4}, z_{5}\right)=z_{4}^{2}+z_{5}^{2}$ and $f(v)=\left(1, v, v^{2}\right)$.

\section{Submanifolds with Gauss rank 2.}

In this section, we will study the structure of $r=2$ case in some more detail.

Let $\iota: M^{n} \rightarrow \mathbf{C}^{N}$ be a complete holomorphic immersion, with Gauss rank $r=2$. Assume $M$ is not an $\mathcal{L}$ cylinder. Then by Corollary 1 in the last section, we know that the Zariski open subset $M^{\prime \prime \prime}$ in $M$ is foliated by the holomorphic foliation $\mathcal{K}=\mathcal{R}$ whose leaves (under $\iota$ ) are open subsets of linear subvarieties of dimension $n-1$. The following completeness result is due to Vitter ([V], Theorem B) and Dajczer-Gromoll ([D-G]).

Proposition 4. For a non-cylinder, complete holomorphic immersion $\iota$ : $M^{n} \rightarrow \mathbf{C}^{N}$ with $r=2$, the leaves of $\mathcal{K}=\mathcal{R}$ are complete. 
We omit the proof here, since it can be found in [D-G] (Proposition 2.1 ), where the authors proved the completeness of $\mathcal{K}$ for complete isometric immersion into $\mathbf{R}^{N}$ with Gauss rank 2. Their result is stronger since it covers the $C^{\infty}$ case. The proof can be easily adapted to the complex case.

Proposition 4 will be used only in this section.

Proposition 5. Any non-cylinder, complete holomorphic immersion $\iota$ : $M^{n} \rightarrow \mathbf{C}^{N}$ with $r=2$ is the total space of a holomorphic fiber bundle $\pi: M^{n} \rightarrow S$ over a Riemann surface $S$. Each fiber of $\pi$ is identified by $\iota$ with a linear subvariety $\mathbf{C}^{n-1}$ in $\mathbf{C}^{N}$.

Proof. First let us extend the holomorphic foliation $\mathcal{K}$ in $M^{\prime \prime \prime}$ into a holomorphic foliation on the entire $M$. Fix any point $x \in M \backslash M^{\prime \prime \prime}$. Let $U$ be a small neighborhood of $x$ such that $\left.\iota\right|_{U}$ is an embedding. We will identify $U$ with its image in $\mathbf{C}^{N}$. Since $M^{\prime \prime \prime}$ is dense in $M$, and $\mathcal{K}$ is a ruling foliation, so for any sequence $\left\{x_{i}\right\}$ in $U \cap M^{\prime \prime \prime}$ approaching $x$, there will be a subsequence $\left\{x_{k_{i}}\right\}$ such that the linear subvarieties containing $\mathcal{K}_{x_{k_{i}}}$ converges to a limit position $P$, which is a linear subvariety of dimension $n-1$ passing through $x$. We claim that this limit position is unique at $x$. Assume the contrary, we will have two sequences in $U \cap M^{\prime \prime \prime},\left\{x_{i}\right\} \rightarrow x$ and $\left\{x_{j}^{\prime}\right\} \rightarrow x$, such that $\mathcal{K}_{x_{i}}$ converges to $P$ and $\mathcal{K}_{x_{j}^{\prime}}$ converges to $P^{\prime}$, with $P \neq P^{\prime}$. By Proposition 4, each $\mathcal{K}_{x_{i}}$ or $\mathcal{K}_{x_{j}^{\prime}}$ is a translate of a $\mathbf{C}^{n-1}$, and hence so is $P$ or $P^{\prime}$. Because $r=2$, we have $n \geq 3$. Consequently, the fact that $\operatorname{dim} P=\operatorname{dim} P^{\prime}=n-1$ implies that $P \cap P^{\prime} \neq \emptyset$ for dimensional reasons. Denote by $\pi$ the restriction on $U$ of the orthogonal projection from $\mathbf{C}^{N}$ to $T_{x} M$, it is a biholomorphism when $U$ is sufficiently small. Since $\pi$ is (the restriction of) a linear map, for $i, j$ sufficiently large, the linear subvarieties $\pi\left(\mathcal{K}_{x_{i}}\right)$ and $\pi\left(\mathcal{K}_{x_{j}^{\prime}}\right)$ will intersect in $\pi(U)$. That is, $\mathcal{K}_{x_{i}}$ will intersect $\mathcal{K}_{x_{j}^{\prime}}$ in $U$, which is impossible. Since holomorphicity follows from the continuity here, we have a holomorphic foliation $\mathcal{K}^{\prime}$ on $M$ which extends $\mathcal{K}$, and the leaves of $\mathcal{K}^{\prime}$ are complete $(n-1)$ dimensional linear subvarieties.

In order to see the bundle structure, let us consider the holomorphic map

$$
\Psi: M \rightarrow G r_{\mathbf{C}}\left(\mathbf{C P}^{n-1}, \mathbf{C P}^{N}\right) \cong G r_{\mathbf{C}}(n, N+1)
$$

which sends $x \in M$ to the $\mathbf{C P}^{n-1}$ containing $\iota\left(\mathcal{K}_{x}^{\prime}\right)$. Denote by $Y$ the image $\Psi(M)$. let $\left\{U_{k}\right\}_{k=1}^{\infty}$ be a countable open covering of $M$ such that each $U_{k}$ is the union of (complete) leaves of $\mathcal{K}^{\prime}$ which is biholomorphic to a direct product $C_{k} \times \mathcal{K}_{x}^{\prime}$, where $C_{k}$ is a nonsingular holomorphic curve transversal to 
$\mathcal{K}^{\prime}$ and $\mathcal{K}_{x}^{\prime}$ is a leaf of $\mathcal{K}^{\prime}$ in $U_{k}$, and $\Psi$ is injective on each $C_{k}$. By shrinking $U_{k}$ if necessary, we may further require that $\Psi\left(U_{k}\right)$ lies in a coordinate neighborhood of $\operatorname{Gr}_{\mathbf{C}}(n, N+1)$. Let $\tilde{S}$ be the quotient space of the union $\bigcup_{k}\left\{C_{k}\right\}$, where the equivalence relation is: $x_{k} \in C_{k}$ is equivalent to $x_{j} \in C_{j}$ iff $x_{k}$ and $x_{j}$ belong to the same leaf of $\mathcal{K}^{\prime} . \tilde{S}$ has an obvious topology that makes it a Hausdorff space. Note that each $\Psi\left(U_{k}\right)$ is a subvariety germ of euclidean space at each of its points. Thus by pulling back the sheaf of germs of holomorphic functions on $\Psi\left(U_{k}\right)$ to $U_{k}, \tilde{S}$ acquires the structure of a 1-dimensional analytic space. Let $S$ be its normalization. Now there is a natural map $\tilde{S} \rightarrow Y$ that sends the equivalence class $[y]$ of $y \in M$ to $\Psi(y)$. The map $\Psi: M \rightarrow Y$ lifts to a map $\Psi: M \rightarrow \tilde{S}$, which in turn lifts to a map $\Psi: M \rightarrow S$. It is straightforward to verify that the latter is a holomorphic bundle with fibre $\mathbf{C}^{n-1}$.

Next, let us consider the special case when $M$ is a hypersurface and is embedded. Let us construct an example first, which is the abstract form of the complete non-cylinder examples constructed in [W] and [V].

Example 4. Let $S$ be a (complex) plane curve in $\mathbf{C}^{2}=\left\{\left(u_{1}, u_{2}\right)\right\} . S$ is not assumed to be nonsingular or a closed subset in $\mathbf{C}^{2}$. Write $\Omega=u_{1}(S)$. We assume that $\Omega$ is a nonempty open subset of C. Suppose $f: \Omega \rightarrow \mathbf{C}^{n} \backslash\{0\}$ is a holomorphic map. We now associate with the pair $S$ and $f$ a hypersurface $M_{S, f}^{n}$ in $\Omega \times \mathbf{C}^{n} \subseteq \mathbf{C}^{n+1}$ defined by:

$$
M_{S, f}^{n}=\left\{(y, z) \in \Omega \times \mathbf{C}^{n} \mid(y, f(y) \cdot z) \in S\right\}
$$

where $f(y) \cdot z=\sum_{i=1}^{n} f_{i}(y) z_{i}$ is the formal complex dot product.

Write $\pi(y, z)=(y, f(y) \cdot z)$. Then $\pi: M \rightarrow S$ is a holomorphic fiber bundle with fiber $\mathbf{C}^{n-1}$.

Claim (A). Notation and assumption as above, $S$ is a nonsingular plane curve if and only if the hypersurface $M_{S, f}^{n}$ is a nonsingular subvariety of $\mathbf{C}^{n+1}$.

First assume $S$ is nonsingular, and we will prove $M_{S, f}^{n}$ is nonsingular. Let $p_{0} \equiv\left(y_{0}, z_{0}\right) \in \Omega \times \mathbf{C}^{n}$, and we will show that the germ of $M\left(\equiv M_{S, f}^{n}\right)$ at $p_{0}$ is nonsingular. Let $S$ be defined at $\pi\left(p_{0}\right)$ by a local coordinate function $w$, and let $G$ be the function germ at $p_{0}$ in $M$ defined by $G(p)=w(y, f(y) \cdot z)$. Then the zero set of $G$ near $p_{0}$ is the germ of $M$ at $p_{0}$. We claim that the complex gradient $\nabla G \equiv\left(\partial G / \partial z_{1}, \ldots, \partial G / \partial z_{n+1}\right)$ of $G$ is nowhere zero near $p_{0}$, which would prove our assertion. Still using the notation $(y, z)$ to 
represent a point $p$ of $\Omega \times \mathbf{C}^{n}$, we compute:

$$
\nabla G(p) \equiv\left(\frac{\partial w}{\partial u_{1}}(\pi(p))+\frac{\partial w}{\partial u_{2}}(\pi(p))\left(f^{\prime}(y) \cdot z\right), \frac{\partial w}{\partial u_{2}}(\pi(p)) f(y)\right) .
$$

Now $\frac{\partial w}{\partial u_{1}}, \frac{\partial w}{\partial u_{2}}$ do not simultaneously vanish at any point of $S$. So suppose $\frac{\partial w}{\partial u_{2}}(\pi(p)) \neq 0$, then already $\nabla G(p) \neq 0$. If however $\frac{\partial w}{\partial u_{2}}(\pi(p))=0$, then $\frac{\partial w}{\partial u_{1}}(\pi(p)) \neq 0$, so that

$$
\nabla G(p)=\left(\frac{\partial w}{\partial u_{1}}(\pi(p)), 0\right) \neq 0 .
$$

This being true for every $p \in M$, the claim is proved.

Now the converse. Suppose $M$ is nonsingular subvariety, and we must prove that $S$ is a nonsingular plane curve. Suppose not, then $S$ has a singularity at some $\pi\left(p_{0}\right)$ for a $p_{0} \in M$. Let the ideal of the germ of $S$ at $\pi\left(p_{0}\right)$ be generated by a function germ $\varphi$. Consider the function germ $g$ at $p_{0}$ defined by $g(p) \equiv \varphi(y, f(y) \cdot z)$ for $p$ near $p_{0}$. Since $S$ is singular at $\pi\left(p_{0}\right)$, we have $\frac{\partial \varphi}{\partial u_{1}}\left(\pi\left(p_{0}\right)\right)=\frac{\partial \varphi}{\partial u_{2}}\left(\pi\left(p_{0}\right)\right)=0$, but at least one of $\frac{\partial \varphi}{\partial u_{1}}(\pi(p))$ and $\frac{\partial \varphi}{\partial u_{1}}(\pi(p))$ is nonzero for any $\pi(p)$ of $S$ near $\pi\left(p_{0}\right)$ but $p \neq p_{0}$. Because $M$ is locally defined by a coordinate function $\zeta$ and $g$ vanishes exactly on $M, g=g_{0} \zeta^{k}$ for some integer $k \geq 1$ and for some holomorphic function $g_{0}$ vanishing nowhere on the germ of $M$ at $p_{0}$. Hence on the germ of $M$ at $p_{0}$, $\nabla g$ is either nowhere zero $(k=1)$ or identically zero $(k>1)$. But

$$
\nabla g(p)=\left(\frac{\partial \varphi}{\partial u_{1}}(\pi(p))+\frac{\partial \varphi}{\partial u_{2}}(\pi(p))\left(f^{\prime}(y) \cdot z\right), \frac{\partial \varphi}{\partial u_{2}}(\pi(p)) f(y)\right) .
$$

So if for one $p \in M, \frac{\partial \varphi}{\partial u_{2}}(\pi(p)) \neq 0$, the fact that $f(y) \neq 0$ implies that $\nabla g(p) \neq 0$ and hence $\nabla g$ is nowhere zero on $M$ near $p_{0}$. In particular, $\nabla g\left(p_{0}\right) \neq 0$. However, the fact that $\frac{\partial \varphi}{\partial u_{1}}\left(\pi\left(p_{0}\right)\right)=\frac{\partial \varphi}{\partial u_{2}}\left(\pi\left(p_{0}\right)\right)=0$ implies that $\nabla g\left(p_{0}\right)=0$, a contradiction. Hence $\frac{\partial \varphi}{\partial u_{2}}(\pi(p))=0$ for any $p$ on $M$ near $p_{0}$. Take a $p_{1}$ near $p_{0}$ but $\neq p_{0}$. Then $\frac{\partial \varphi}{\partial u_{1}}\left(\pi\left(p_{1}\right)\right) \neq 0(S$ is nonsingular at $\left.p_{1}\right)$. Thus

$$
\nabla g\left(p_{1}\right)=\left(\frac{\partial \varphi}{\partial u_{1}}\left(\pi\left(p_{1}\right)\right), 0\right) \neq 0,
$$

and therefore $\nabla g$ must be nowhere zero near $p_{0}$. In particular, $\nabla g\left(p_{0}\right) \neq 0$, and we have reached the same contradiction as before.

By Claim (A), we know that $M\left(=M_{S, f}^{n}\right)$ is an embedded smooth hypersurface in $\Omega \times \mathbf{C}^{n} \subseteq \mathbf{C}^{n+1}$. Define $\rho(f)=\operatorname{dim} \operatorname{span}\{f(y) \mid y \in \Omega\} . \rho(f)$ 
is the dimension of the minimal subspace of $\mathbf{C}^{n}$ containing $f(\Omega)$. We can now produce developable submanifolds:

Claim (B). Let $S$ be a nonsingular plane curve and let the hypersurface $M_{S, f}^{n}$ be complete.

(i) If $\rho(f)=1$, then $M_{S, f}^{n}$ is a cylinder of Gauss rank $r \leq 1$.

(ii) If $\rho(f)=2$, then $M_{S, f}^{n}$ is a cylinder of Gauss rank $r=2$.

(iii) If $\rho(f) \geq 3, M_{S, f}^{n}$ is a developable submanifold with Gauss rank $r=2$ but is not a cylinder.

For any $p_{0} \equiv\left(y_{0}, z_{0}\right) \in M$, let $V_{p_{0}}=\left\{\left(y_{0}, z_{0}+v\right) \mid v \in \mathbf{C}^{n}, f\left(y_{0}\right) \cdot v=\right.$ $\left.f^{\prime}\left(y_{0}\right) \cdot v=0\right\}$. Note that $V_{p_{0}} \subseteq M$, and $\pi\left(V_{p_{0}}\right)=\pi\left(p_{0}\right)$.

Suppose $\rho(f)=1$, then $f\left(y_{0}\right)$ is proportional to $f^{\prime}\left(y_{0}\right)$ and therfore $V_{p_{0}}$ is an $(n-1)$-dimensional linear subvariety passing through $p_{0}$. We now prove that $\nabla G$ is constant on $V_{p_{0}}$. As before, let $S$ be defined at $\pi\left(p_{0}\right)$ by a local coordinate function $w$, and let $G$ be the function germ at $p_{0}$ in $M$ defined by $G(p)=w(y, f(y) \cdot z)$. Then the zero set of $G$ near $p_{0}$ is the germ of $M$ at $p_{0}$. We see that the complex gradient $\nabla G \equiv\left(\partial G / \partial z_{1}, \ldots, \partial G / \partial z_{n+1}\right)$ of $G$ is

$$
\nabla G(p) \equiv\left(\frac{\partial w}{\partial u_{1}}(\pi(p))+\frac{\partial w}{\partial u_{2}}(\pi(p))\left(f^{\prime}(y) \cdot z\right), \frac{\partial w}{\partial u_{2}}(\pi(p)) f(y)\right) .
$$

It follows immediately from this formula that if $p_{0}=\left(y_{0}, z_{0}\right)$ as above and $p=\left(y_{0}, z_{0}+v\right) \in V_{p_{0}}$, then

$$
\begin{aligned}
\nabla G(p) & =\left(\frac{\partial w}{\partial u_{1}}(\pi(p))+\frac{\partial w}{\partial u_{2}}(\pi(p))\left(f^{\prime}\left(y_{0}\right) \cdot z_{0}\right), \frac{\partial w}{\partial u_{2}}(\pi(p)) f\left(y_{0}\right)\right) \\
& =\nabla G\left(p_{0}\right),
\end{aligned}
$$

so that $\nabla G$ is constant on $V_{p_{0}}$ for each such $p_{0}$. Since the tangent plane of $M$ at $p$ is defined by $p+\left\{w \in \mathbf{C}^{n+1} \mid \nabla G(p) \cdot w=0\right\}$, the tangent planes of $M$ are constant along $V_{p_{0}}$, as claimed. Hence the Gauss rank $r \leq 1$. By Abe's theorem ([A]; see Theorem 4 of [W] for a different proof), $M$ is a cylinder.

Suppose $\rho(f)=2$. Because $f \wedge f^{\prime}$ is not identically zero, for all but a discrete set of $y_{0}$ 's, the set $\left\{v \in \mathbf{C}^{n} \mid f\left(y_{0}\right) \cdot v=f^{\prime}\left(y_{0}\right) \cdot v=0\right\}$ is an $(n-2)$ dimensional subspace of $\mathbf{C}^{n}$. Thus there is a Zariski open set of $p_{0}$ 's for which $V_{p_{0}}$ is an $(n-2)$-dimensional linear subvariety passing through $p_{0}$. The fact that the tangent space of $M$ is constant along $V_{p_{0}}$ for all such $p_{0}$ is proved in 
the same way as before. This proves that $r \leq 2$. To show $r=2$, it suffices to show that $r=1$ leads to a contradiction. If $r=1$, the same theorem of Abe shows that $M$ is a cylinder with $(n-1)$-dimensional rulings. Thus (with notation as above) the complex gradient $\nabla G$ is orthogonal to $(n-1)$ orthonormal vectors $W_{1}, W_{2}, \ldots, W_{n-1}$. Write $W_{i}=\left(\omega_{i}, W_{i}^{\prime}\right) \in \Omega \times \mathbf{C}^{n}$ as usual. We claim that at least one $\omega_{i} \neq 0$. If not, the fact that $\left\langle\nabla G, W_{i}\right\rangle=0$ for all $i$ (where $\langle$,$\rangle denots the ordinary Hermitian inner product on \mathbf{C}^{n+1}$ ) implies that

$$
\frac{\partial w}{\partial u_{2}}(\pi(p))\left\langle f(y), w_{i}^{\prime}\right\rangle=0
$$

for all $i$. Thus the linear span of $f(\Omega)$ is orthogonal to the $(n-1)$-dimensional linear span of $w_{1}, w_{2}, \ldots, w_{n-1}$ in $\mathbf{C}^{n}$. But $\rho(f)=2$ means that the linear span of $f(\Omega)$ has dimension 2, and this is impossible for dimensional reasons. So let us say $\omega_{1} \neq 0$. For simplicity of notation, let us write $\omega$ for $\omega_{1}, W^{\prime}$ for $W_{1}^{\prime}$, and $W$ for $W_{1}$, so that $W=\left(\omega, W^{\prime}\right)$ and $\langle\nabla G(p), W\rangle=0$ for all $p \in \Omega \times \mathbf{C}^{n}$. Writing $p=(y, z)$, we have that for all $p \in M$ :

$$
\begin{aligned}
0= & \langle\nabla G(p), W\rangle \\
= & \left\{\frac{\partial w}{\partial u_{1}}(\pi(p))+\frac{\partial w}{\partial u_{2}}(\pi(p))\left(f^{\prime}(y) \cdot z\right)\right\} \bar{\omega} \\
& +\frac{\partial w}{\partial u_{2}}(\pi(p))\left\langle f(y), W^{\prime}\right\rangle .
\end{aligned}
$$

Now let $p_{0}=\left(y_{0}, z_{0}\right)$ as before. We have seen that $\frac{\partial w}{\partial u_{2}}\left(\pi\left(p_{0}\right)\right) \neq 0$ for a Zariski open set of $p_{0}$ 's in $M$. For such a $p_{0}$, let $v^{\prime} \in \mathbf{C}^{n}$ so that $f\left(y_{0}\right) \cdot v^{\prime}=0$ but $f^{\prime}\left(y_{0}\right) \cdot v^{\prime} \neq 0$. Define $p=\left(y_{0}, z_{0}+t v^{\prime}\right)$ where $t$ is an arbitrary complex number for the moment. Note that $\pi(p)=\pi\left(p_{0}\right)$ because $f\left(y_{0}\right) \cdot v^{\prime}=0$. So with this choice of $p$, we have:

$$
\begin{aligned}
0= & \langle\nabla G(p), W\rangle \\
= & \left\{\frac{\partial w}{\partial u_{1}}\left(\pi\left(p_{0}\right)+\frac{\partial w}{\partial u_{2}}\left(\pi\left(p_{0}\right)\right)\left(f^{\prime}\left(y_{0}\right) \cdot z_{0}+t f^{\prime}\left(y_{0}\right) \cdot v^{\prime}\right)\right)\right\} \bar{\omega} \\
& +\frac{\partial w}{\partial u_{2}}\left(\pi\left(p_{0}\right)\right)\left\langle f(y), W^{\prime}\right\rangle \\
= & \left\{\frac { \partial w } { \partial u _ { 1 } } \left(\pi\left(p_{0}\right)+\frac{\partial w}{\partial u_{2}}\left(\pi\left(p_{0}\right)\right)\left(f^{\prime}\left(y_{0}\right) \cdot z_{0}\right\} \bar{\omega}\right.\right. \\
& +t\left\{\frac{\partial w}{\partial u_{2}}\left(\pi\left(p_{0}\right)\right)\left(f^{\prime}\left(y_{0}\right) \cdot v^{\prime}\right)\right\} \bar{\omega}+\frac{\partial F}{\partial u_{2}}\left(\pi\left(p_{0}\right)\right)\left\langle f(y), W^{\prime}\right\rangle,
\end{aligned}
$$

for any choice of $t \in \mathbf{C}$. Because $\left\{\frac{\partial F}{\partial u_{2}}\left(\pi\left(p_{0}\right)\right)\left(f^{\prime}\left(y_{0}\right) \cdot v^{\prime}\right)\right\} \bar{\omega} \neq 0$ by choice, this is impossible. Therefore $r \neq 1$, and necessarily $r=2$. 
It remains to see that $M$ is a cylinder. For each $p=(y, z) \in \Omega \times \mathbf{C}^{n}$, let $\mathcal{S}_{p} \equiv \operatorname{span}\left\{\overline{f(y)}, \overline{f^{\prime}(y)}\right\}$. Then (observing that $f(y) \cdot z=\langle z, \overline{f(y)}\rangle$ ) each $V_{p}$ is an $(n-2)$-dimensional linear subvariety in $\mathbf{C}^{n}$ orthogonal to $\mathcal{S}_{p}$. We have observed that for a Zariski open subset $M_{0}$ of $M, \operatorname{dim} \mathcal{S}_{p}=2$ for all $p \in M_{0}$. .. Now let $\mathcal{S} \equiv \operatorname{span}\{\overline{f(y)} \mid y \in \Omega\}$. Clearly, $\mathcal{S}_{p} \subseteq \mathcal{S}$ for all $p \in M$. By hypothesis, $\operatorname{dim} \mathcal{S}=2$. Thus for dimensional reasons, $\mathcal{S}_{p}=\mathcal{S}$ for all $p \in M_{0}$. It follows that if $\mathcal{S}^{\perp}$ denotes the orthogonal complement $\left\{v \in \mathbf{C}^{n} \mid\langle v, \mathcal{S}\rangle=0\right\}$ of $\mathcal{S}$ in $\mathbf{C}^{n}$, then for every $p \in M_{0}, V_{p}$ is just the linear subvariety $p+\left(0, \mathcal{S}^{\perp}\right)$. In particular, all the $V_{p}$ 's are parallel and therefore $M$ is a cylinder, as claimed. This completes the proof of case (ii).

Finally, let $\rho(f) \geq 3$. Then as in case (ii), each $V_{p_{0}}$ is an $(n-2)$ dimensional linear subvariety orthogonal to $\mathcal{S}_{p_{0}}$ for all $p_{0}$ in a Zariski open subset $M_{0}$ of $M$, and the Gauss rank of $r=2$. To see that $M$ cannot be a cylinder in the present situation, consider two points $p_{1}$ and $p_{2}$

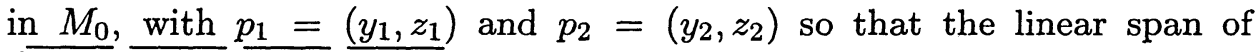
$\left\{\overline{f\left(y_{1}\right)}, \overline{f^{\prime}\left(y_{1}\right)}, \overline{f\left(y_{2}\right)}, \overline{f^{\prime}\left(y_{2}\right)}\right\}$ has dimension $\geq 3$. The subspaces $\mathcal{S}_{p_{1}}$ and $\mathcal{S}_{p_{2}}$ being distinct 2-dimensional subspaces of $\mathbf{C}^{n}$, their orthogonal complements $\mathcal{S}_{p_{1}}^{\perp}$ and $\mathcal{S}_{p_{2}}^{\perp}$ (defined as in the preceding paragraph) are no longer parallel, and therfore the linear subvarieties $V_{p_{1}}$ and $V_{p_{2}}$ are also not parallel to each other. So $M$ is not a cylinder.

In the Introduction, we defined a hypersurface $M_{0}=\left\{x_{4}^{2} x_{1}+\left(x_{4}-1\right) x_{2}+\right.$ $\left.\left(x_{4}-2\right) x_{3}=0\right\}$ in $\mathbf{C}^{4}$. In our present notation, $M_{0}$ can be alternatively described as follows. In $\mathbf{C}^{2}$, the Riemann surface $S$ is defined by $u_{2}=0$ so that $S$ is just the $u_{1}$-axis and $\Omega=\mathbf{C}$. The function $f: \mathbf{C} \rightarrow \mathbf{C}^{3} \backslash\{0\}$ is now given by $f(y)=\left(y^{2}, y-1, y-2\right)$, so that $M$ is given in $\mathbf{C}^{4}$ by $f(y) \cdot z=0$, i.e., $y^{2} z_{1}+(y-1) z_{2}+(y-2) z_{3}=0$.

We would like to remark that in Example 4, $S$ does not have to be a closed subset in $\mathbf{C}^{2}$ even when $M^{n}$ is closed in $\mathbf{C}^{n+1}$. For example, consider $\Omega=\mathbf{C} \backslash\{0\}$,

$$
S=\left\{\left(y, e^{y}\right) \mid y \in \Omega\right\} \subset \mathbf{C}^{2}
$$

and let $f: \Omega \rightarrow \mathbf{C}^{3} \backslash\{0\}$ be the map defined by $f(y)=\left(y, y^{2}, y^{3}\right)$. Then

$$
M_{S, f}^{3}=\left\{\left(y, z_{1}, z_{2}, z_{3}\right) \in \mathbf{C}^{4} \mid y\left(z_{1}+y z_{2}+y^{2} z_{3}\right)=e^{y}\right\}
$$

is closed in $\mathbf{C}^{4}$, while $S$ is not closed in $\mathbf{C}^{2}$.

Theorem 3. Let $M^{n} \subseteq \mathbf{C}^{n+1}$ be a complete embedded hypersurface with Gauss rank $r=2$. Assume that $M$ is not a cylinder, then there exists $S$ and $f$ as in Example 4 so that $M=M_{S, f}^{n}$. 
Proof. It follows from Theorem 2 and Proposition 4 that the leaves $\mathcal{K}_{x}$ of $\mathcal{K}$ are $(n-1)$ planes. The key point of the proof is that, under the codimension 1 and embeddedness assumption, these $(n-1)$ planes will have a common normal vector in $\mathbf{C}^{n+1}$. In order to see this, let us fix a generic point $x$ on $M$. Under congruence let us assume that $x=0$ is the origin in $\mathbf{C}^{n+1}$ and $M$ is locally given by

$$
z_{n+1}=h\left(z_{1}, \ldots, z_{n}\right)
$$

with $\mathcal{L}_{0}=\operatorname{span}\left\{e_{1}, \ldots, e_{n-2}\right\}, \mathcal{K}_{0}=\operatorname{span}\left\{e_{1}, \ldots, e_{n-1}\right\}$, and $T_{0} M=$ $\operatorname{span}\left\{e_{1}, \ldots, e_{n}\right\}$, where $e_{i}=\frac{\partial}{\partial z_{i}}$. So $h(0)=0, d h(0)=0$.

Consider the curve $C(s) \stackrel{=}{=}(0, \ldots, 0, s, h(0, \ldots 0, s))$ in $M,|s|<\epsilon$. By the definition of $\mathcal{L}$ and $\mathcal{K}=\mathcal{R}$, there exists holomorphic vector fields $\xi^{i}(s)$ $(1 \leq i \leq n-2)$ along $C(s)$ such that $\mathcal{L}_{C(s)}$ and $\mathcal{K}_{C(s)}$ are spanned by $\left\{\xi^{i} ; 1 \leq i \leq n-2\right\}$ and $\left\{\xi^{i}, \xi^{i \prime} ; 1 \leq i \leq n-2\right\}$, respectively, where $\xi^{i \prime}$ denotes $d \xi^{i} / d s$. Without loss of generality, we may assume $\xi^{1} \wedge \ldots \wedge \xi^{n-2} \wedge \xi^{1 \prime} \neq 0$ at $s=0$. Under the choice of our coordinates,

$$
\begin{aligned}
\mathcal{K}_{C(0)} & =\mathbf{C}\left\{e_{1}, \ldots, e_{n-1}\right\} \subseteq M^{n} \\
\mathcal{K}_{C(s)} & =C(s)+\mathbf{C}\left\{\xi^{1 \prime}, \xi^{1}, \ldots, \xi^{n-2}\right\}=\{F(s, t)\}_{t \in \mathbf{C}^{n-1}} \quad \text { where } \\
F(s, t) & =C(s)+\sum_{i=1}^{n-2} t_{i} \xi^{i}(s)+t_{n-1} \xi^{1 \prime}(s) .
\end{aligned}
$$

The last two components of $F(s, t)$ are

$$
\begin{aligned}
F_{n} & =s+\sum_{i=1}^{n-2} t_{i} \xi_{n}^{i}(s)+t_{n-1} \xi_{n}^{1 \prime}(s) \\
F_{n+1} & =h(0, \ldots, 0, s)+\sum_{i=1}^{n-2} t_{i} \xi_{n+1}^{i}(s)+t_{n-1} \xi_{n+1}^{1 \prime}(s) .
\end{aligned}
$$

Now we claim that the two $(n-1)$ holomorphic vectors $\left(\xi_{n}^{1}, \ldots, \xi_{n}^{n-2}, \xi_{n}^{1 \prime}\right)$ and $\left(\xi_{n+1}^{1}, \ldots, \xi_{n+1}^{n-2}, \xi_{n+1}^{1 \prime}\right)$ are always parallel to each other for any $|s|<\epsilon$.

Assume the contrary, that is, their wedge is not identically zero. Then for any generic point $s$ in the punctured $\epsilon$-disc, there will be some $t \in \mathbf{C}^{n-1}$ such that $F_{n}=F_{n+1}=0$, that is $\mathcal{K}_{C(0)} \cap \mathcal{K}_{C(s)} \neq \phi$, which contradicts Proposition 5.

Now we know that $\left(\xi_{n}^{1}, \ldots, \xi_{n}^{n-2}, \xi_{n}^{1 \prime}\right)$ is parallel to $\left(\xi_{n+1}^{1}, \ldots, \xi_{n+1}^{n-2}, \xi_{n+1}^{1 \prime}\right)$ for any small $s$. This implies that there exist constants $a$ and $b$, not both zero, such that

$$
\begin{array}{rll}
a \xi_{n}^{i}(s)+b \xi_{n+1}^{i}(s)= & \quad \forall i, \quad \forall|s|<\epsilon \\
a \xi_{n}^{1 \prime}(s)+b \xi_{n+1}^{1}(s)=0 & \forall|s|<\epsilon .
\end{array}
$$


Because $\xi^{i \prime} \in \operatorname{span}\left\{\xi^{1 \prime}, \xi^{1}, \ldots, \xi^{n-2}\right\}$ for any $i$, it follows that for any small $s, \mathcal{K}_{C(s)}$ is perpendicular to (the complex conjugate of) $-b e_{n}+a e_{n+1}$. By analyticity, we know that all the leaves of $\mathcal{K}$ are perpendicular to a fixed direction in $\mathbf{C}^{n+1}$. (Since $M$ is assumed to be non-cylinder, such a direction must be unique.)

Now if we rotate our coordinate $\left\{z_{i}\right\}$, we may assume that this common normal direction for $\mathcal{K}$ is just $e_{1}$. Let $\Omega=z_{1}(M) \subseteq \mathbf{C}$. For each constant $c$, the intersection $M_{c}=\left\{z_{1}=c\right\} \cap M^{n}$ is a disjoint union of parallel $(n-1)$ dimensional linear subvarieties, which are leaves of $\mathcal{K}$. Write $V_{c} \equiv \mathbf{C}^{n-1}$ for the linear subspace in $\mathbf{C}^{n}=\left\{z_{1}=0\right\}$ that is parallel to the linear subvarieties in $M_{c}$.

Denote points in $\mathbf{C}^{n+1}$ as $\left(z_{1}, z^{\prime}\right)$, where $z^{\prime} \equiv\left(z_{2}, \ldots, z_{n+1}\right)$. Define a map $g: \Omega \rightarrow \mathbf{C P}^{n-1}$ as follows. If $c \in \Omega$, let $V_{c}$ be defined in $\mathbf{C}^{n}=$ $\left\{z_{1}=0\right\}$ by $c_{2} z_{2}+\ldots+c_{n+1} z_{n+1}=0$ for some constants $c_{2}, \ldots, c_{n+1}$. Then by definition, $g(c)=\left[\begin{array}{llll}c_{2} & : \ldots & : c_{n+1}\end{array}\right]$ (homogeneous coordinates). $g$ is clearly holomorphic. Since $\Omega \subseteq \mathbf{C}$, by the Weierstrass theorem, there will be a global holomorphic map $f: \Omega \rightarrow \mathbf{C}^{n} \backslash\{0\}$ that lifts $g$, that is, if $f(u)=\left(a_{1}, \ldots a_{n}\right)$, then $g(u)=\left[a_{1}: \ldots: a_{n}\right]$.

Now consider the complex plane curve $S$ in $\mathbf{C}^{2}$ defined by:

$$
S=\left\{\left(u, f(u) \cdot z^{\prime}\right) \mid u \in \Omega,\left(u, z^{\prime}\right) \in M\right\} .
$$

It is also clear that, for this $S$ and $f, M$ is just the hypersurface $M_{S, f}^{n}$ defined in Example 4. (Note that because of Claim (A), $S$ is a nonsingular plane curve, and because of Claim (B) and the hypothesis that $M$ is not a cylinder, $\rho(f) \geq 3$.) This completes the proof of Theorem 3 .

Remark. A semi-global version of Theorem 3 was due to Vitter ([V], Theorem $\mathrm{C})$.

The codimension 1 condition is obviously necessary in Theorem 3 , as illustrated by the following example.

Example 5. Consider $F: \mathbf{C}^{3} \rightarrow \mathbf{C}^{5}$ defined by $F\left(s, t_{1}, t_{2}\right)=\psi(s)+$ $t_{1} \xi^{\prime}(s)+t_{2} \xi(s)$, where

$$
\psi=(0,0,0,0, s), \quad \xi=\left(1, s, s^{2}, s^{3}, h(s)\right)
$$

with $h(s)$ an arbitrary holomorphic function on $\mathbf{C}$. It is easy to check that $F$ is indeed an embedding, that the leaves of the Gauss foliation are the $t_{2}$-curves, and that the conullity foliation $\mathcal{K}$ is the obvious one, namely, if $x=F\left(s, t_{1}, t_{2}\right)$ and $V_{x}=\operatorname{span}\left\{\xi^{\prime}(s), \xi(s)\right\}$, then $\mathcal{K}_{x}=x+V_{x}$. Clearly, there 
is no direction in $\mathbf{C}^{4}$ that is perpendicular to all the leaves of $\mathcal{K}$ so that $F(\mathbf{C})$ can never be represented as an $M_{S, f}^{3}$.

The embeddedness condition in Theorem 3 is also necessary for the conclusion to hold. Consider the following example:

Example 6. Suppose $S$ is any non-compact Riemann surface, $\xi, \psi$ are holomorphic maps from $S$ into $\mathbf{C}^{4}$ such that for any $s \in S, \quad\left(\psi^{\prime} \wedge \xi \wedge \xi^{\prime} \wedge\right.$ $\left.\xi^{\prime \prime}\right)(s) \neq 0$. (This condition is obviously independent of the choice of local holomorphic coordinate at $s$ ). Then the holomorphic map $F: S \times \mathbf{C}^{2} \rightarrow \mathrm{C}^{4}$ defined by $F=\psi+t_{1} \xi^{\prime}+t_{2} \xi$ is an immersion. When $\xi$ is non-degenerate (i.e., $\xi(S)$ does not lie in any proper linear subvariety), the leaves of $\mathcal{K}$ (which are the translates of the subspaces $\operatorname{span}\left\{\xi^{\prime}(s), \xi(s)\right\}$ ) do not have a common normal direction. So again, $F(S \times \mathbf{C})$ can never be an $M_{S, f}^{3}$.

For a specific example, one can take $S=\mathbf{C}, \psi=\xi^{\prime \prime}$ and $\xi=\left(1, s, s^{2}, s^{3}\right)$. In this case, $F\left(s+\frac{1}{\delta},-6 \delta, 12 \delta^{2}\right) \equiv F\left(s, 6 \delta, 12 \delta^{2}\right)$ for any $s \in \mathbf{C}$ and any $\delta \in$ $\mathbf{C}^{*}$. In particular, $F$ is not proper. In fact, if we replace the embeddedness condition in Theorem 3 by requiring the hypersurface $M$ to be properly immersed, then the leaves of $\mathcal{K}$ again will have a common normal direction, and one gets a similar (but slightly more complicated) description as in Theorem 3. We will omit the details here.

Remark. In the real case, in [D-G], Dajczer and Gromoll were interested in this $r=2$ class from the point of view of isometric deformations. In view of Theorem 1 of this paper, it would be very interesting to explore the structural results for complete, non-cylinder developable submanifolds of low Gauss ranks, e.g., $\mathrm{r}=3$ or 4 , both in the real and the complex cases.

\section{Topology.}

In this section, we will discuss the topological aspect of developable submanifolds. We would like to raise the following question (topological cylinder conjecture):

Question 1. Suppose $\left(M^{n}, \iota\right)$ is a proper holomorphic immersion in $\mathbf{C}^{N}$ with Gauss rank $r<n$. Is it true that $M^{n}$ is always biholomorphic to a cylinder $X^{r} \times \mathbf{C}^{n-r}$ for some Stein manifold $X^{r}$ ?

Towards this direction, we observe that, using the proof of AndreottiFrankel to the Lefschetz hyperplane section theorem ([A-F]), the homotopy type of such a manifold $M^{n}$ is that of a $C W$ complex of dimension $r$ or less: 
Proposition 6. A properly immersed developable complex submanifold $\left(M^{n}, \iota\right)$ in $\mathbf{C}^{N}$ of Gauss rank $r<n$ is homotopy equivalent to a $C W$ complex of dimension $\leq r$.

Proof. Let $\mathcal{S}=M \backslash M^{\prime}$ be the singular set of the Gauss map $\Gamma$. Pick a generic point $p$ in $\mathbf{C}^{N}$, so that $p$ is not on $\iota(M)$, not a focal point of $\iota(M)$, and not in the normal space $\mathcal{N}_{\iota(x)}$ for any $x \in \mathcal{S}$. Then the square of the distance from $p$ to $\iota(M)$ induces a positive proper exhaustion function on $M$, which is a Morse function. At any critical point $q \in M$ of this function, using the defining property of $\mathcal{L}_{q}$ via second fundamental form, the same proof in [A-F] yields that index of $q$ must be less then or equal to $r$.

It is worth noting that the above result does not hold for non-developable ruled submanifolds. For instance, consider the smooth quadric $M^{2}=\left\{z_{1}^{2}+\right.$ $\left.z_{2}^{2}+z_{3}^{2}=1\right\} \subseteq \mathbf{C}^{3}$. It is foliated by straight complex lines. But its second betti number $b_{2}=1+b_{1}>0$, since its Euler number $e=1-b_{1}+b_{2}=4-2=2$ as $M$ is the complement of the diagonal line in $\mathbf{C P}^{1} \times \mathbf{C P}^{1}$. (Another way to look at it is, $M^{2}$ is a holomorphic line bundle over $\mathbf{C P}^{1}$.)

Question 2. Suppose $\left(M^{n}, \iota\right)$ is a proper holomorphic immersion in $\mathbf{C}^{N}$ with Gauss rank $r<n$. If it is not a cylinder, is it true that $H_{r}(M, \mathbf{Z})=0$ ? Is this true at least when $M$ is a properly embeded hypersurface?

By Proposition 5, this is the case for $r=2$. The reasoning here is that, intuitively, when $M$ is further and further away from being a cylinder, its topology seems to be more and more restrictive.

If we push Question 1 one step further, we may even ask the following

Question 3. Suppose $M^{n}$ is a properly embedded complex submanifold in $\mathbf{C}^{N}$ with Gauss rank $r<n$. Is it true that there always exists a continuous path $\gamma:[0,1] \rightarrow \operatorname{Aut}\left(\mathbf{C}^{N}\right)$ in the (holomorphic) automorphism group such that $\gamma(0)$ is the identity, while $\gamma(1)$ maps $M^{n}$ onto a cylinder $N^{n}=\mathbf{C}^{n-r} \times X^{r}$ and carries the Gauss leaves of $M$ onto those $\mathbf{C}^{n-r}$ in $N$ ? In other words, even though $M$ is not isometricly a cylinder, can it be deformed (by ambient automorphisms) into a cylinder? If so, what is the smallest subgroup of $\operatorname{Aut}\left(\mathbf{C}^{N}\right)$ within which this can be done?

We hope these questions and the general discussion can generate some interest towards this special class of submanifolds. In particular, we believe that the structure of the singular set $\mathcal{S}=M \backslash M^{\prime}$ of the Gauss map should be analyzed and it should contain a lot of information about $M$. 
Finally, let us close our discussion with the following observation, which is the direct consequence of Theorem 7 in $[\mathrm{A}]$ - to the effect that for a developable submanifold $N$ in $\mathbf{C}^{n}$, if the holomorphic curvature of a plane orthogonal to a leave of the Gauss foliation is always nonzero at one point, then $N$ is a cylinder, - together with the fact that, on a non-positively curved Kähler manifold, the vanishing of the holomorphic sectional curvature in a direction implies the vanishing of the Ricci curvature in that direction (cf. [Z]).

Theorem 4. Suppose $\left(M^{n}, \iota\right)$ is a complete immersed complex submanifold in $\mathbf{C}^{N}$ with Gauss rank $r<n$. If for some complex Euclidean metric $d s^{2}$ on $\mathbf{C}^{N}$, the pull back Kähler metric $\iota^{*}\left(d s^{2}\right)$ has non-positive sectional curvature, then $M$ is a cylinder, that is, there exists complete holomorphic immersion $\iota^{\prime}: X^{r} \rightarrow \mathbf{C}^{N-n+r}$ such that $\iota=\iota^{\prime} \times i d_{\mathbf{C}^{n-r}}$.

Proof. Let $x \in M^{\prime}$. By Theorem 7 of [A], it suffices to show that the holomorphic sectional curvatures $R_{Y \bar{Y} Y \bar{Y}}^{M} \neq 0$ for any $Y \in \mathcal{L}_{x}^{\perp}$, the orthogonal complement of $\mathcal{L}_{x}$ in $T_{x} M$. Assume that $R_{Y \bar{Y} Y \bar{Y}}^{M}=0$ for some $Y \in \mathcal{L}_{x}^{\perp}$. For any $Z \in T_{x} M$ not parallel to $Y$, consider the complex plane spanned by $Y$ and $Z$. By [Z], $R_{Y \bar{Y} Z \bar{Z}}^{M}=0$. Since $Z$ is arbitrary, the Ricci curvature in $Y$ is also zero, which contradicts the fact that $\mathcal{L}_{x}=\left\{X \in T_{x} M \mid \operatorname{Ricci}_{X \bar{X}}=0\right\}$.

\section{References.}

[A] K. Abe, Applications of a Riccati type differential equation to Riemannian manifolds with totally geodesic distributions, Tôhoku Math. J., 25 (1973), 425-444.

[A-F] A. Andreotti and T. Frankel, The Lefschetz theorem on hyperplane sections, Ann. of Math., 69 (1959), 713-717.

[C-K] S.-S. Chern and N. Kuiper, Some theorems on the isometric imbedding of compact Riemannian manifolds in Euclidean space, Ann. of Math., 56 (1952), 422-430.

[D-G] M. Dajczer and D. Gromoll, Rigidity of complete Euclidean hypersurfaces, J. Differential Geom., 31 (1990), 401-416. 
[D-R] M. Dajczer and L. Rodriquez, Complete real Kähler minimal submanifolds, J. Reine Angew. Math., 419 (1991), 1-8.

[F] D. Ferus, On the completeness of nullity foliations, Michigan Math. J., 18 (1971), 61-64.

[F-W] G. Fischer and $\mathrm{H}$. Wu, Developable complex analytic submanifolds, International J. Math., 6 (1995), 229-272.

[G-H] P.A. Griffiths and J. Harris, Algebraic geometry and local differential geometry, Ann. Ec. Norm. Sup., 12 (1979), 355-423.

[V] A. Vitter, Twisted-cylinder theorem for complex submanifolds, preprint, 1979.

[W] $\mathrm{H} . \mathrm{Wu}$, Complete developable submanifolds in real and complex Euclidean spaces, International J. Math., 6 (1995), 461-489.

[Z] F. Zheng, First Pontrjagin form, rigidity and strong rigidity of nonpositively curved Kähler surfaces, Math. Z., 220 (1995), 159-169.

Department of Mathematics \#3840

UNIVERSITY OF CALIFORNIA

BERKELEY, CA 94720-3840

E-mail address: wu@math.berkeley.edu

DEPARTMENT OF MATHEMATICS

OHIO STATE UNIVERSITY

Columbus, OH 43210-1174

E-mail address: zheng@math.ohio-state.edu

Received August 31, 2000. 